\title{
A study of bright southern slowly pulsating B stars *
}

\section{The intrinsic frequencies ${ }^{\star \star}$}

\begin{abstract}
P. De Cat ${ }^{\star \star \star}$ and C. Aerts
Instituut voor Sterrenkunde, Katholieke Universiteit Leuven, Celestijnenlaan 200 B, 3001 Leuven, Belgium

Received 7 May 2002/ Accepted 19 July 2002

Abstract. We present the results of detailed frequency analyses of a sample of thirteen confirmed slowly pulsating B stars. Our analysis is based on a combination of elaborate photometric and spectroscopic data-sets. The original sample consists of a mixture of five confirmed slowly pulsating B stars and twelve candidate slowly pulsating B stars discovered thanks to the photometric measurements of the HIPPARCOS satellite. HD 55522 and HD 131120 turn out to be chemically peculiar stars. HD 169978 and HD 69144 are two ellipsoidal variables for which no intrinsic variability is found. At least nine of the thirteen studied slowly pulsating B stars are multi-periodic. For HD 74195, HD 85953, HD 123515 and HD 215573, the observed frequency spacings suggest that we are dealing with frequency multiplets. For the apparent mono-periodic binary HD 24587, it is not clear if the observed variations are induced by stellar pulsation and/or by rotation modulation. We highlight the statistical character of the observed pulsational properties of our sample.
\end{abstract}

Key words. stars: early-type - stars : variables: general - stars: oscillations - binaries: spectroscopic - line: profiles

\section{Introduction}

The term "slowly pulsating B stars" (hereafter "SPBs") was introduced for a distinct group of mid-B type photometric variables pulsating in high-radial-order $g$-modes with periods of the order of days (Waelkens 1991). The SPBs have several members in common with the 53 Per stars which were defined as a group of spectroscopic variables surrounding the $\beta$ Cep stars (Smith 1977). The observed variations of the SPBs are understood in terms of the $\kappa$ mechanism (e.g. Dziembowski et al. 1993). Thanks to the space-based photometric measurements of the ESA mission HIPPARCOS, the number of SPBs was enlarged from 12 to about 100 (Waelkens et al. 1998).

Aerts et al. (1999) selected a sample of 17 southern stars for long-term monitoring to study the behaviour of the $g$ mode pulsations of SPBs. This sample contains 5 previously known SPBs (hereafter "confirmed SPBs") and 12 new candidate SPBs discovered thanks to the photometric measurements of the HIPPARCOS satellite (hereafter "HIPPARCOS

\section{Send offprint requests to: P. De Cat,}

e-mail: Peter.DeCat@ster.kuleuven.ac.be

* Based on observations collected with the CAT Telescope of the European Southern Observatory and with the Swiss Photometric Telescope of the Geneva Observatory, both situated at La Silla in Chile

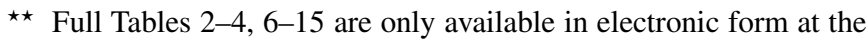
CDS via anounymous ftp to

cdsarc.u-strasbg.fr $(130.79 .128 .5)$ or via

http://cdsweb.u-strasbg.fr/cgi-bin/qcat?]/A+A/393/965

$\star \star \star$ Postdoctoral Fellow of the Fund for Scientific Research, Flanders.
SPBs”). The Si II-doublet centered at $4130 \AA$ was preferred to study the occurring line-profile variations. The 7 filters of the Geneva photometric system are used for the study of their light variations. During some 20 observation campaigns between March 1996 and March 1999, ground-based follow-up measurements were gathered with respectively the Coudé Auxiliary Telescope/Coudé Echelle Spectrometer (CAT/CES) combination and the Swiss Telescope/Geneva P7 photometer of the Geneva Observatory, both situated at La Silla (Chile).

This series of papers is devoted to the detailed analysis of the final time-series of the targets in Aerts et al. (1999). In the first paper (De Cat et al. 2000, hereafter referred to as Paper I), the orbital parameters and the main intrinsic frequency of the spectroscopic binaries among the sample were determined. For a full description of the observations and data reductions, we refer to Sect. 2 of that paper. This paper is devoted to the determination of all the intrinsic frequencies contained in the data of both the binary and single targets. For modes with a sufficiently high amplitude we are also performing mode identification, the results of which will be discussed in a separate forthcoming paper (De Cat \& Aerts 2002, in preparation).

This paper is organised as follows. We start with an overview of the general properties of the targets in Sect. 2. In Sect. 3, we describe the methods used for the analyses. The results of the binary and single stars are discussed respectively in Sects. 4 and 5. In Sect. 6, an overview of the pulsational properties of this sample of bright southern SPBs is given. We end with our conclusions and a discussion of our results in Sect. 7. 
Table 1. The spectral type (SpT), the effective temperature $T_{\text {eff }}$ (in $\mathrm{K}$ ), gravity $\log g$ (in cgs units), $\operatorname{luminosity} \log L_{1}$ (in $L_{\odot}$ ), mass $M_{1}$ (in $M_{\odot}$ ), radius $R_{1}$ (in $R_{\odot}$ ), bolometric magnitude $M_{\mathrm{bol}}$, and distance $d$ (in parsec) of the targets in the sample of Aerts et al. (1999). The errors are given between brackets in units of the last decimal. The confirmed SPBs known before the HIPPARCOS mission are marked with $*$, the new spectroscopic binary with $\bullet$, and the chemically peculiar stars with $\circ$.

\begin{tabular}{|c|c|c|c|c|c|c|c|c|c|c|c|}
\hline & & star & & SpT & $T_{\mathrm{eff}}$ & $\log g$ & $\log L_{1}$ & $M_{1}$ & $R_{1}$ & $M_{\mathrm{bol}}$ & $d$ \\
\hline \multirow[t]{2}{*}{$\mathrm{SB} 2 e \neq 0$} & $*$ & HD 123515 & HIP 69174 & B9IV & $11990(60)$ & $4.27(9)$ & $1.91(9)$ & $3.0(1)$ & $2.1(2)$ & $-0.02(23)$ & 168(19) \\
\hline & & HD 140873 & HIP 77227 & B8III & $13930(80)$ & $4.35(12)$ & $2.17(12)$ & $3.6(2)$ & $2.1(3)$ & $-0.67(30)$ & $125(11)$ \\
\hline \multirow[t]{4}{*}{$\mathrm{SB} 1 e \neq 0$} & & HD 24587 & HIP 18216 & B5V & $13860(70)$ & $4.26(12)$ & $2.27(12)$ & $3.7(2)$ & $2.4(3)$ & $-0.91(30)$ & $118(10)$ \\
\hline & $\bullet$ & HD 53921 & HIP 34000 & B9IV & $13690(70)$ & $4.23(12)$ & $2.28(12)$ & $3.7(2)$ & $2.4(3)$ & $-0.93(30)$ & $148(12)$ \\
\hline & $*$ & HD 74560 & HIP 42726 & B3IV & $16210(150)$ & $4.15(14)$ & $2.77(14)$ & $4.9(2)$ & $3.1(5)$ & $-2.16(35)$ & $147(10)$ \\
\hline & $*$ & HD 177863 & HIP 93887 & B8V & $13380(60)$ & $4.14(12)$ & $2.33(12)$ & $3.7(2)$ & $2.7(4)$ & $-1.06(30)$ & $222(52)$ \\
\hline \multirow[t]{3}{*}{$\mathrm{SB} 1 e=0$} & & HD 92287 & HIP 52043 & B3IV & $16420(140)$ & $4.00(15)$ & $2.98(15)$ & $5.3(4)$ & $3.8(7)$ & $-2.69(38)$ & $392(78)$ \\
\hline & & HD 69144 & HIP 40285 & B2.5IV & $15880(120)$ & $3.80(16)$ & $3.15(16)$ & $5.6(5)$ & 4.9(9) & $-3.10(40)$ & $298(50)$ \\
\hline & & HD 169978 & HIP 90797 & B7.5III & $12760(60)$ & $3.86(13)$ & $2.55(13)$ & $3.9(3)$ & $3.9(6)$ & $-1.61(33)$ & $147(16)$ \\
\hline \multirow[t]{8}{*}{ Single } & $*$ & HD 74195 & HIP 42536 & B3IV & $16160(130)$ & $3.91(16)$ & $3.05(16)$ & $5.4(4)$ & $4.3(8)$ & $-2.87(40)$ & $152(12)$ \\
\hline & $*$ & HD 181558 & HIP 95159 & B5III & $14680(90)$ & $4.16(13)$ & $2.52(13)$ & $4.2(2)$ & $2.8(4)$ & $-1.54(33)$ & $208(36)$ \\
\hline & & HD 26326 & HIP 19398 & B5IV & $15210(110)$ & $4.14(14)$ & $2.63(14)$ & $4.4(2)$ & $3.0(5)$ & $-1.82(35)$ & $223(39)$ \\
\hline & & HD 85953 & HIP 48527 & B2III & $18440(180)$ & $3.91(17)$ & $3.37(17)$ & $6.6(5)$ & $4.7(10)$ & $-3.66(43)$ & $524(140)$ \\
\hline & & HD 138764 & HIP 76243 & B6IV & $14050(80)$ & $4.20(12)$ & $2.37(12)$ & $3.9(1)$ & $2.6(4)$ & $-1.16(30)$ & $108(10)$ \\
\hline & & HD 215573 & HIP 112781 & B6IV & $13960(80)$ & $4.09(13)$ & $2.48(13)$ & $4.0(2)$ & $3.0(5)$ & $-1.45(33)$ & $136(9)$ \\
\hline & $\circ$ & HD 55522 & HIP 34798 & B2IV/V & $17430(160)$ & $4.15(15)$ & $2.95(15)$ & $5.5(3)$ & $3.3(6)$ & $-2.61(38)$ & $220(30)$ \\
\hline & $\circ$ & HD 131120 & HIP 72800 & B7IIIp & $18250(190)$ & $4.13(15)$ & $3.09(15)$ & $6.0(3)$ & $3.5(6)$ & $-2.95(38)$ & $118(11)$ \\
\hline
\end{tabular}

\section{General properties}

Before starting the analysis, we carried out a thorough inspection of all the data-sets. We only retained HIPPARCOS measurements with a quality flag 0 . Geneva measurements with a colour quality label $p=0$ and/or a $V$ magnitude quality label $q=0$ were removed from the original data-sets. Spectra with $S / N<100$ were not considered during the analyses. Finally, all observations with values deviating more than $3 \sigma$ from the mean value $\mu$ of the considered data-set were removed (after having checked that we are not dealing with eclipses in the case of photometric data-sets). The number of removed observations is always less than $10 \%$ of the number of retained ones.

The resulting Geneva data-sets were used to determine the effective temperature $T_{\text {eff }}$, gravity $\log g$, luminosity $\log L_{1}$, radius $R_{1}$, and bolometric magnitude $M_{\text {bol }}$ of the spectroscopic binaries in the sample of Aerts et al. (1999) in the same way as described by Waelkens et al. (1998). The spectral type SpT and the HIPPARCOS distance $d$ are also given in Table 1 . These values should be preferred to those listed in Aerts et al. (1999). From here on, error estimates are given between brackets in units of the last decimal (when available).

The observed equivalent width (hereafter " $E W$ ") of spectral absorption lines are extremely sensitive to the temperature of the considered star. In Fig. 1, the $E W$ values of the $4128 \AA$ $\mathrm{Si}$ II line as a function of the effective temperature are compared with theoretical values from Didelon (1982). There are four targets for which the average equivalent width of the observed Si II profiles significantly deviates from those of other targets with a similar effective temperature. On the one hand, the observed $E W$ values of HD 53921 and HD 24587 are relatively low (Fig. 1, open circles). These stars therefore deserve special attention. On the other hand, the observed $E W$ values

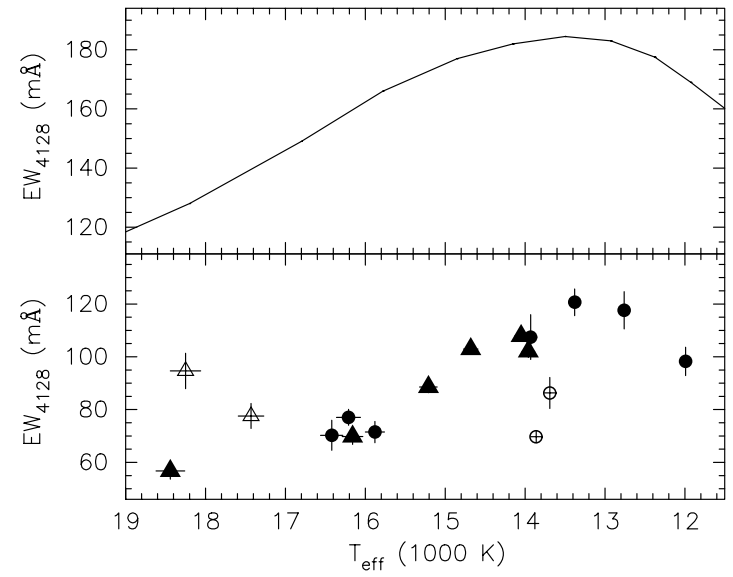

Fig. 1. Top: The equivalent width $E W(\AA)$ of the $4128 \AA$ Si II line as a function of effective temperature for stars with luminosity classes IIIIV-V (data taken from Didelon 1982). Bottom: The observed average equivalent width $E W(\mathrm{~m} \AA$ ) of the $4128 \AA \mathrm{Si}$ II line of the targets in the sample of Aerts et al. (1999) as a function of their effective temperature $T_{\text {eff }}(\mathrm{K})$ as given in Table 1. The error bars denote the standard errors of the observed properties. The spectroscopic binaries and the single stars are respectively given by circles and triangles. The targets that do not follow the general trend (the binaries HD 53921 and HD 24587, and the single stars HD 55522 and HD 131120) are represented by open symbols.

of HD 55522 and HD 131120 are relatively high (Fig. 1, open triangles). Both targets turn out to be chemically peculiar stars whose variations are caused by a non-homogeneous distribution of the elements on the stellar surface. These results will not be presented here. For a full discussion, we refer to Briquet et al. (2001) and De Cat (2001). 
HD 69144 and HD 169978 are two spectroscopic binaries for which no main intrinsic period could be found in our datasets (Paper I). However, HD 69144 exhibits clear line-profile variations pointing towards stellar pulsation. We need additional higher-temporal resolution data for an in-depth analysis of the variability of this star. HD 169978 turns out to be a nonpulsating, ellipsoidal variable, which was misclassified as an SPB. These two targets will not be discussed any more.

\section{Used methods}

\subsection{Mass/inclination procedure}

The radial velocities derived from the Si II profiles were already used in Paper I for the determination of the orbits of the spectroscopic binaries. The derived orbital elements and estimations for $M_{1}$ and $R_{1}$ are used here to put constraints on the orbital inclination angle $i$ and the mass $M_{2}$ of the secondary by using what we will call the "mass/inclination procedure". For each choice of $i, M_{2}$ is derived from the mass function $f(M)$. Subsequently, the radius $R_{2}$ of a main-sequence secondary is estimated and herefrom the semi-major axis $a_{1,2}$ of the relative orbit. In some cases, restrictions on $i$, and hence on $M_{2}$ can be found by expressing that the periastron distance has to be larger than the sum of the two radii $R_{1}+R_{2}$. Moreover, the absence of eclipses and the single-lined character provide us an upper limit of respectively $i$ and $M_{2}$. Note that for double-lined binaries, exact values of $i$ and $M_{2}$ can be derived when an estimation of $M_{1}$ is available.

\subsection{Frequency procedure}

We used what we will call the "frequency procedure" to determine the intrinsic frequencies in the final data-sets. We calculated the first three normalised velocity moments $\langle v\rangle,\left\langle v^{2}\right\rangle$, and $\left\langle v^{3}\right\rangle$ of the individual Si II lines (see Aerts et al. 1992). During analysis, we generally focussed on the moments of the deepest Si II line, except if blending effects occurred. Since we are dealing with B stars, we focussed on the $B$-filter of the Geneva photometric system. For every star, we subsequently analysed data-sets with increasing frequency resolution $\Delta v_{\text {win }}$, which we define as the half-width at half-max (HWHM) of the central peak of the window function.

Three frequency search algorithms were combined: Stellingwerf's PDM-algorithm (Stellingwerf 1978), the modified Scargle periodogram (Horne \& Baliunas 1986), and the CLEAN-algorithm (Roberts et al. 1987). We restrict ourselves to discuss the modified Scargle periodograms in the text below. To judge upon the reality of the different occurring frequency peaks in the periodograms, we used both the "false alarm probability" (FAP, Scargle 1981) and a " $S / N$-criterion" (Breger et al. 1993). The noise-level of a periodogram is empirically defined as the average amplitude in an oversampled spectrum surrounding the suspected frequency, where the sampling interval is $\Delta v=\frac{1}{20 T}$ with $T$ the total time-span of the considered data-set ${ }^{1}$ (Handler et al. 1996). Alvarez et al. (1998) showed that the $99 \%$ confidence level can be reproduced fairly well by taking 3.7 times the noise-level for multi-site data of p-mode pulsators. To check its applicability to single-site data of $g$-mode pulsators, the amplitude-level corresponding to a FAP of $1 \%$ and to 3.7 times the noise-level (hereafter " $3.7 \mathrm{~S} / \mathrm{N}$ level") are indicated respectively by a dashed and a dotted line on every shown modified Scargle periodogram.

If more than one intrinsic frequency is present in the data, we used subsequent prewhitening with sinusoidal models. The frequency analysis is stopped when all the frequency peaks are below the $1 \%$ FAP-level and/or the $3.7 \mathrm{~S} / \mathrm{N}$-level and/or when we were not able to distinguish between different candidate frequencies. The residual standard deviation $\sigma_{\text {res }}$ is compared to the level of mean error of the observations $\sigma_{N}$ to judge upon the possibility of having more intrinsic frequencies than the ones retained from our analysis.

Afterwards, the observed amplitudes $A_{i}$, phases $\phi_{i}$, and their corresponding standard errors, are determined for all the accepted frequencies $v_{i}$ by fitting the data with a superposition of sinusoidal models of the form $A_{i} \sin \left[2 \pi\left(v_{i} t+\phi_{i}\right)\right]$, where $t=$ HJD - 2450000. For the higher order moments, the appropriate interaction terms are also taken into account (see Mathias et al. 1994). In the following, $A_{U}, \ldots, A_{G}$ denote the observed photometric amplitudes of respectively the Geneva $U, \ldots, G$ data (same notations as in Aerts 2000), $A_{\mathrm{H}_{\mathrm{p}}}$ the observed photometric amplitude of the HIPPARCOS $H_{\mathrm{p}}$ data, and $\{A A\},\{C C, D D\}$ and $\{E E, F F, R S T\}$ the observed moment amplitudes of respectively the $\langle v\rangle,\left\langle v^{2}\right\rangle$ and $\left\langle v^{3}\right\rangle$ data (same notations as in Aerts 1996).

\section{Binary SPBs}

In the following three subsections, we present the results of the mass/inclination and frequency procedure for the binaries.

\subsection{Double-lined binaries}

\subsubsection{HD 123515 - HR 5296 - HIP 69174}

HD $123515\left(m_{V}=5.96\right)$ is double-lined spectroscopic binary with $P_{\text {orb }}=26.036(4) \mathrm{d}$ and $e=0.264(7)$ (Paper I). With our spectra, we derive a mass-ratio of $M_{2} / M_{1}=0.629(8)$, and hence $M_{2} \approx 1.9(1) M_{\odot}$ and $i \approx 54^{\circ}$. All these observations are compatible with a main-sequence A 6 component.

HD 123515 is one of the 7 SPB-prototypes introduced by Waelkens (1991) for which he found 4 intrinsic photometric frequencies: $v_{1, \mathrm{p}}=0.68521 \mathrm{c} \mathrm{d}^{-1}, v_{2, \mathrm{p}}=0.65928 \mathrm{c} \mathrm{d}^{-1}$, $v_{3, p}=0.72861 \mathrm{~cd}^{-1}$, and $v_{4, \mathrm{p}}=0.45834 \mathrm{~cd}^{-1}$. With our data, also 4 intrinsic frequencies are found: $v_{1}=0.68528(10) \mathrm{c} \mathrm{d}^{-1}$, $v_{2}=0.65929(10) \mathrm{cd}^{-1}, v_{3}=0.72585(10) \mathrm{cd}^{-1}$, and $v_{4}=$ $0.55198(10) \mathrm{cd}^{-1} \cdot v_{1, \mathrm{p}}$ and $v_{2, \mathrm{p}}$ correspond to $v_{1}$ and $v_{2}$ while $v_{3, p}$ and $v_{4, p}$ are aliases of respectively $v_{3}$ and $v_{4}$. The modified Scargle periodograms and the best theoretical sinusoidal fits to the data after subsequent prewhitening are shown

\footnotetext{
1 The Geneva photometric data-sets generally consist of a few old measurements besides new ones gathered during our dedicated SPBruns. Both groups of measurements are separated by several years. In these cases, we used the total time-span $T^{*}$ of the dedicated SPB-runs instead of the total time-span $T$ of all measurements.
} 


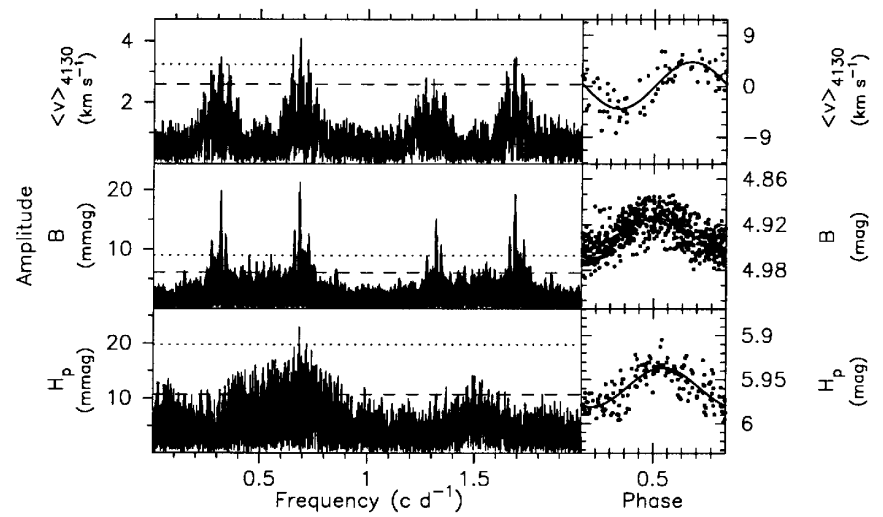

Table 2. Overview of the characteristics of the accepted intrinsic frequencies in the different data-sets of HD 123515. For the meaning of the different symbols, we refer to the text. The amplitudes for which 0 is in the asymptotic $95 \%$ confidence interval are given in italic. The full table for all colours and velocity moments is only available in electronic at the CDS via anonymous ftp to cdsarc.u-strasbg.fr (130.79.125.5) or via http://cdsweb.u-strasbg.fr/cgi-bin/qcat?J/A+A/393/965

\begin{tabular}{|c|c|c|c|c|c|}
\hline data-set & $\sigma$ & $A_{i}$ & $\phi_{i}$ & $\sigma_{\text {res }}$ & $\sigma_{N}$ \\
\hline \multicolumn{6}{|c|}{$v_{1}=0.68528(10) \mathrm{c} \mathrm{d}^{-1}$} \\
\hline$<v>_{4130}\left(\mathrm{~km} \mathrm{~s}^{-1}\right)$ & 3.69 & $3.54(34)$ & $0.50(2)$ & & \\
\hline$B \quad(\mathrm{mmag})$ & 22.3 & $19.6(6)$ & $0.241(5)$ & & \\
\hline$H_{\mathrm{p}} \quad(\mathrm{mmag})$ & 21.2 & $20.9(12)$ & $0.203(9)$ & & \\
\hline \multicolumn{6}{|c|}{$v_{2}=0.65929(10) \mathrm{c} \mathrm{d}^{-1}$} \\
\hline$B \quad(\mathrm{mmag})$ & & $13.6(5)$ & $0.875(7)$ & & \\
\hline (mmag) & & $10.9(13)$ & $0.819(21)$ & & \\
\hline \multicolumn{6}{|c|}{$v_{3}=0.72585(10) \mathrm{c} \mathrm{d}^{-1}$} \\
\hline$<v>_{4130}\left(\mathrm{~km} \mathrm{~s}^{-1}\right)$ & & $1.91(36)$ & $0.15(3)$ & 1.93 & 0.54 \\
\hline$B \quad(\mathrm{mmag})$ & & $11.0(6)$ & $0.967(8)$ & & \\
\hline$H_{\mathrm{p}} \quad(\mathrm{mmag})$ & & $11.9(11)$ & $0.947(18)$ & & \\
\hline \multicolumn{6}{|c|}{$v_{4}=0.55198(10) \mathrm{c} \mathrm{d}^{-1}$} \\
\hline$B \quad$ (mmag) & & $6.1(6)$ & $0.43(1)$ & 10.1 & \\
\hline$H_{\mathrm{p}} \quad(\mathrm{mmag})$ & & $7.8(12)$ & $0.25(3)$ & 9.4 & 6.2 \\
\hline
\end{tabular}

only the case for $v_{1}$. The 4 frequencies are all present in the $H_{\mathrm{p}}$ variations, but they are found in a different order $\left(v_{1}, v_{3}, v_{2}\right.$, $\left.v_{4}\right)$. Without the Geneva data, we would not have been able to pin-point the physical frequencies from the different candidate frequencies with an amplitude exceeding the $1 \%$ FAP-level. In the spectroscopic variations, no evidence for $v_{2}$ or $v_{4}$ is found. After prewhitening the Geneva data with $v_{1}, v_{2}, v_{3}$, and $v_{4}$, there are still several frequencies whose amplitude exceeds both significance levels, of which $v_{5}=0.68817(10) \mathrm{c} \mathrm{d}^{-1}$ is one of the best candidates. However, there is no reason to prefer this candidate to another. Therefore, $v_{5}$ will not be considered during the modelling.

The amplitudes and phases for the fits with the four accepted intrinsic frequencies are given in Table 2, together with the original standard deviation $\sigma$, the residual standard deviation $\sigma_{\text {res }}$, and the level of mean error of the observations $\sigma_{N}$ (when available) of the different data-sets. Here, only the properties of the accepted intrinsic frequencies in the $\langle v\rangle$, Geneva $B$ and HIPPARCOS $H_{\mathrm{p}}$ data are given. The properties in the other velocity moments and the other Geneva filters are additionally given in the full table, which is only available electronically. The observed frequency spacings between $v_{1}, v_{2}, v_{3}$ and $v_{4}$ are small, which may point towards membership of a frequency multiplet. The frequency splitting induced by the effect of stellar rotation on high-order $g$-mode pulsations is, in a good approximation, given by:

$$
\begin{aligned}
v_{l, m} \simeq & v_{l, 0}-m\left(1-\frac{1}{l(l+1)}\right) v_{\mathrm{rot}} \\
& +\frac{m^{2} v_{\mathrm{rot}}^{2}}{v_{l, 0}} \frac{4 l(l+1)[2 l(l+1)-3]-9}{2 l^{2}(l+1)^{2}[4 l(l+1)-3]}+\theta\left(v_{\mathrm{rot}}^{3}\right)
\end{aligned}
$$




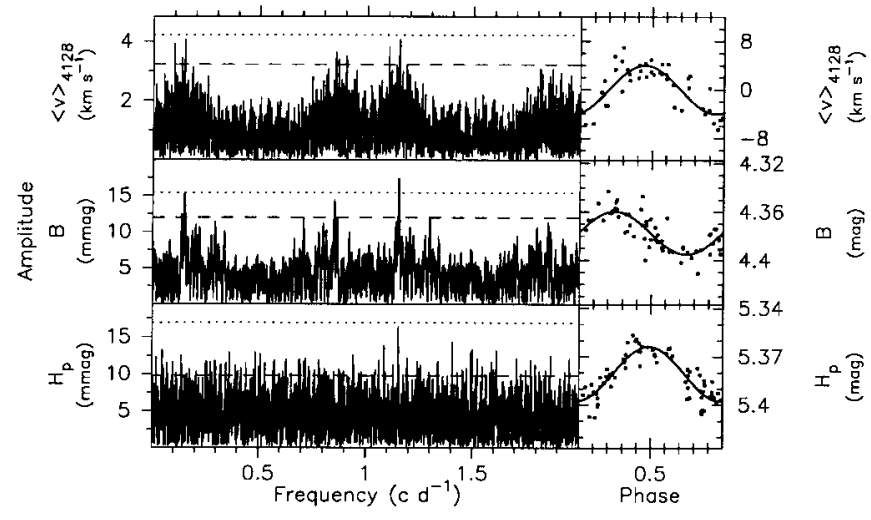

Fig. 3. Same as Fig. 2, but for the accepted intrinsic frequency $v_{1}=$ $1.1515(8) \mathrm{cd}^{-1}$ of HD 140873.

(Dziembowski \& Goode 1992), where $v_{l, 0}$ denotes the pulsation frequency in the corotating frame, $v_{l, m}$ the observed pulsation frequency, and $v_{\text {rot }}$ the rotation frequency of the star. If we assume synchronisation $\left(v_{\text {orb }}=v_{\text {rot }}=0.038410 \mathrm{c} \mathrm{d}^{-1}\right)$, we find an equatorial rotation velocity of some $4 \mathrm{~km} \mathrm{~s}^{-1}$ and the observed frequency spacings between $v_{1}, v_{2}, v_{3}$ and $v_{4}$ would be compatible with a septuplet structure. The overal line broadening is indeed small for the star (Aerts et al. 1999) and the pulsational broadening is relatively large. Hence, such a rotation velocity is feasible. However, we cannot take this suggestion of a septuplet for granted as the orbit is still quite eccentric and the separation between the stars is too large for tidal effects to be very efficient.

\subsubsection{HD 140873 - HR 5863 - HIP 77227}

In Paper I we showed that HD $140873\left(m_{V}=6.24\right)$ is a double-lined spectroscopic binary with $P_{\text {orb }}=38.927(4) \mathrm{d}$ and $e=0.731(6)$. We derive a mass-ratio of $M_{2} / M_{1}=0.50(1)$, and hence $M_{2} \approx 1.8(1) M_{\odot}$ and $i \approx 53^{\circ}$. These observations are compatible with an A $7 \mathrm{~V}$ secondary.

In the past, HD 140873 has been used as a standard star for different photometric systems (e.g. Taylor 1986; Paunzen et al. 1997). However, from our data-sets it is clear that we are dealing with an intrinsic variable. For most of our data-sets of HD 140873, the two highest peaks in the output of the frequency search algorithms correspond to $v_{1}^{\prime}=0.1487(8) \mathrm{c} \mathrm{d}^{-1}$ and $v_{1}=1.1515(8) \mathrm{cd}^{-1}$ (Fig. 3). The $3.7 \mathrm{~S} / \mathrm{N}$-level is only exceeded in the Geneva data. $v_{1}^{\prime}$ and $v_{1}$ are each others $1 \mathrm{~cd}^{-1}$ alias frequency, but $v_{1}$ is the intrinsic one since only evidence for $v_{1}$ is found in the $H_{\mathrm{p}}$ variations. Although there are still some peaks reaching the $1 \%$ FAP-level in the $H_{\mathrm{p}}$ data and in some filters of the Geneva data after prewhitening with $v_{1}$, the current data-sets are insufficient to unambiguously designate additional intrinsic frequencies. In Table 3, we give an overview of the characteristics of $v_{1}$.
Table 3. Same as Table 2, but for the accepted intrinsic frequency of HD 140873.

\begin{tabular}{ccccccc}
\hline \hline \multicolumn{2}{c}{ data-set } & $\sigma$ & $A_{i}$ & $\phi_{i}$ & $\sigma_{\text {res }}$ & $\sigma_{N}$ \\
\hline \multicolumn{2}{c}{$v_{1}=1.1515(8) \mathrm{c} \mathrm{d}^{-1}$} & & & & & \\
$\langle v\rangle_{4128}$ & $\left(\mathrm{~km} \mathrm{~s}^{-1}\right)$ & 3.62 & $4.02(47)$ & $0.80(2)$ & 2.14 & 2.0 \\
$B$ & $(\mathrm{mmag})$ & 15.4 & $17.7(19)$ & $0.51(2)$ & 9.5 & \\
$H_{\mathrm{p}}$ & $(\mathrm{mmag})$ & 13.4 & $16.8(12)$ & $0.27(1)$ & 6.6 & 5.1 \\
\hline
\end{tabular}

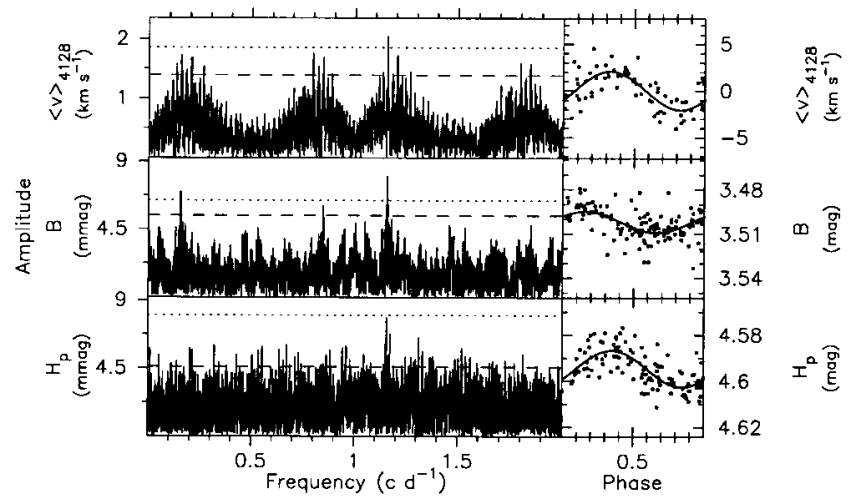

Fig. 4. Same as Fig. 2, but for the accepted intrinsic frequency $v_{1}=$ $1.1569(6) \mathrm{c} \mathrm{d}^{-1}$ of HD 24587.

\subsection{Single-lined binaries with eccentric orbits}

\subsubsection{HD 24587 - HR 1213 - HIP 18216}

The "mass/inclination" procedure applied to the eccentric 459(4) day orbit (see Paper I) of HD $24587\left(m_{V}=4.63\right)$ leads to a lower limit of approximately 2.6 $M_{\odot}$ for $M_{2}$. If the secondary is a main-sequence star, this would imply that we are dealing with an early A-type companion. However, synthetic spectra show that a B $6 \mathrm{~V}+\mathrm{A} 0-2 \mathrm{~V}$ binary system is doublelined, which is not seen in our spectra. The only way out is to assume a neutron star companion, which implies that the system has undergone a phase of mass-transfer through Roche lobe overflow and has survived the supernova explosion.

Mathys et al. (1986) were the first to detect light variations for HD 24587. They found a frequency $0.5786 \mathrm{c} \mathrm{d}^{-1}$ and concluded that the light curves resemble those of many chemically peculiar stars (hereafter "CP stars") whose variations are due to the non-homogeneous distribution of elements on the stellar surface. HD 24587 was listed as a suspected CP star in the "General Catalogue of Ap and Am stars" (Renson et al. 1991). However, the spectroscopic study of Leone \& Catanzaro (1998) led to the conclusion that HD 24587 presents chemical elements which are only slightly underabundant with respect to main sequence stars. Moreover, the observed $E W$ values are constant and consistent with the effective temperature. These observations are confirmed by Catanzaro et al. (1999), who concluded that HD 24587 is not a CP star.

All our data-sets point towards the same intrinsic frequency: $v_{1}=1.1569(6) \mathrm{c} \mathrm{d}^{-1}$ (Fig. 4). The corresponding frequency peaks in the modified Scargle periodograms clearly exceed the 1\% FAP-level and (almost) reach the $3.7 \mathrm{~S} / \mathrm{N}$ level. After prewhitening with $v_{1}$, some peaks still reach the 
Table 4. Same as Table 2, but for the accepted intrinsic frequency of HD 24587.

\begin{tabular}{ccccccc}
\hline \hline \multicolumn{2}{c}{ data-set } & $\sigma$ & $A_{i}$ & $\phi_{i}$ & $\sigma_{\text {res }}$ & $\sigma_{N}$ \\
\hline$v_{1}=1.1569(6) \mathrm{c} \mathrm{d}^{-1}$ & & & & & \\
$\langle v\rangle_{4128}$ & $\left(\mathrm{~km} \mathrm{~s}^{-1}\right)$ & 1.99 & $2.1(2)$ & $0.92(2)$ & 1.37 & 0.50 \\
$\langle v\rangle_{4121}$ & $\left(\mathrm{~km} \mathrm{~s}^{-1}\right)$ & 2.53 & $2.2(3)$ & $0.86(2)$ & 1.98 & \\
$B$ & $(\mathrm{mmag})$ & 10.12 & $7.3(10)$ & $0.59(2)$ & 8.60 & \\
$H_{\mathrm{p}}$ & $(\mathrm{mmag})$ & 7.95 & $7.9(8)$ & $0.40(2)$ & 5.66 & 4.6 \\
\hline
\end{tabular}

$1 \%$ FAP-level in the $H_{\mathrm{p}}$ data and the velocity moments. However, there is no reason to prefer one candidate above the other. Although we cannot clearly identify more than one intrinsic frequency in our data-sets, the remaining standard deviations $\sigma_{\text {res }}$ (Table 4) are still rather high and point towards multi-periodicity. We find no evidence for variations with (sub)harmonics of $v_{1}$. These observations are in favour of the classification of HD 24587 as an SPB. However, $v_{1}$ is twice the frequency found by Mathys et al. (1986) and the observed average $E W$ of the Si II profiles is relatively small compared to those of other target stars with similar temperature (Fig. 1). These observations are in their turn in favour of the earlier classification of HD 24587 as a CP star.

The variability induced by spots can (but need not) be quite different for different spectral lines as far as amplitudes, phases and harmonics are concerned. We therefore additionally studied the time behaviour of the $4121 \AA$ He I profile, which is also situated within in the observed wavelength range. Just like for the moments derived from the Si II profiles, variations with $v_{1}$ are obviously present in $\langle v\rangle$ and $\left\langle v^{3}\right\rangle$, but they are less pronounced in the variations of the $E W$ and $<v^{2}>$. Again, we find no evidence for the appearance of (sub)harmonics of $v_{1}$, nor of other frequencies. The behaviour of the Si II doublet and the He I line is therefore similar, and so does not rule out a pulsation model.

In Table 4, an overview of the characteristics of $v_{1}$ is given. From our data-sets, it is not clear if the observed variations of HD 24587 are induced by stellar pulsation or by rotation modulation, or perhaps both. We are currently performing additional spectroscopic observations to disentangle the variable nature of this star in full detail.

\subsubsection{HD 53921 - HR 2674 - HIP 34000}

HD $53921\left(m_{V}=5.64\right)$ is known as a close visual binary with two B-type components (Corbally 1984). It has never been proven nor ruled out that the visual components are physically bound. This object was listed as a suspected spectroscopic binary in Paper I.

Some additional spectra were taken in November 1998 with the CORALIE spectrograph attached to the Euler telescope situated at La Silla (Chile). The radial velocities and the corresponding weights for the orbital solution determination were obtained from the $\lambda \lambda 4130 \AA$ Si II-doublet in a similar way as for the CAT data (see Paper I). The simultaneous use of the CAT and CORALIE data results in the orbit as shown in the upper panel of Fig. 5. The corresponding parameters are given
Table 5. The orbital parameters for HD 53921. The "Si II" and "4132.5 $\AA$ ” column give the orbit as found in the radial velocities derived from respectively the Si II-doublet and the $4132.5 \AA$ feature.

\begin{tabular}{llcc}
\hline \hline & & Si II & $4132.5 \AA$ \\
\hline$P_{\text {orb }}(\mathrm{d})$ & $=$ & $338(2)$ & $340(3)$ \\
$v_{\gamma}\left(\mathrm{km} \mathrm{s}^{-1}\right)$ & $=$ & $9.9(3)$ & \\
$t(\tau)(\mathrm{HJD})$ & $=$ & $50498(4)$ & $50500(5)$ \\
$e$ & $=$ & $0.63(6)$ & $0.43(8)$ \\
$\omega\left({ }^{\circ}\right)$ & $=$ & $302(15)$ & $283(11)$ \\
$K\left(\mathrm{~km} \mathrm{~s}^{-1}\right)$ & $=$ & $3.2(3)$ & $8.5(4)$ \\
$a \sin i(\mathrm{AU})$ & $=$ & 0.077 & 0.24 \\
$f(M)\left(M_{\odot}\right)$ & $=$ & 0.0005 & 0.016 \\
$\mathrm{rms}\left(\mathrm{km} \mathrm{s}^{-1}\right)$ & $=$ & 1.302 & 0.173 \\
\hline
\end{tabular}

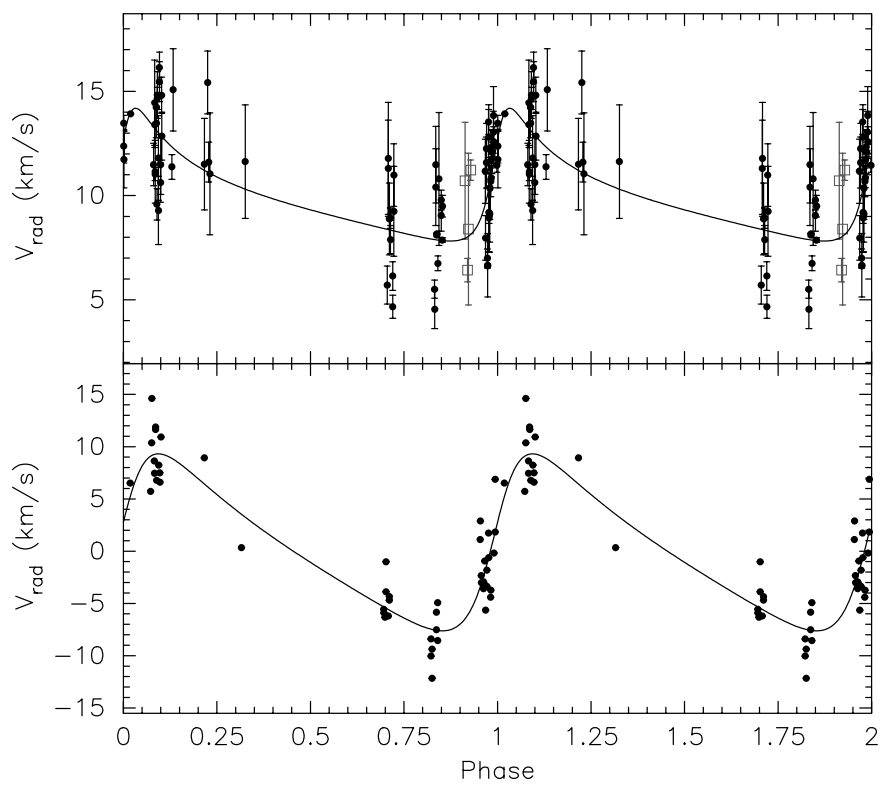

Fig. 5. Observed radial velocities versus orbital phase of HD 53921. The primary component data are given by black dots (CAT data) and grey squares (CORALIE data). The observed radial velocities derived from the observed $\lambda \lambda 4130 \AA$ Si II doublet (top) and the $\lambda \lambda 4132.5 \AA$ feature (bottom) are given together with the theoretical velocities defined by the orbital elements for the primary as given in Table 5 (full lines). In both panels, phase zero corresponds to periastron passage.

in the "Si II" column of Table 5. This orbital solution was tested by considering radial velocities derived from a small unidentified feature near $4132.5 \AA$. These radial velocities were determined by a Gaussian approximation of the line profile after subtraction of the mean central wavelength $4132.583 \AA$. No weights are used. The resulting orbit is shown in the lower panel of Fig. 5. The corresponding parameters are given in the " $4132.5 \AA$ ” column of Table 5. Both sets of orbital parameters are in agreement, except for $e$ and $K$. The $4132.5 \AA$-amplitude is a factor 2.5 larger than the $\mathrm{Si}$ II-amplitude. Since the velocity shifts of the Si II doublet and the $4132.5 \AA$ feature are always in the same direction, they cannot originate from different components of the same spectroscopic binary. We therefore suggest that the observed Si II lines result from the superposition of the Si II lines of both components of the visual binary, and that one 


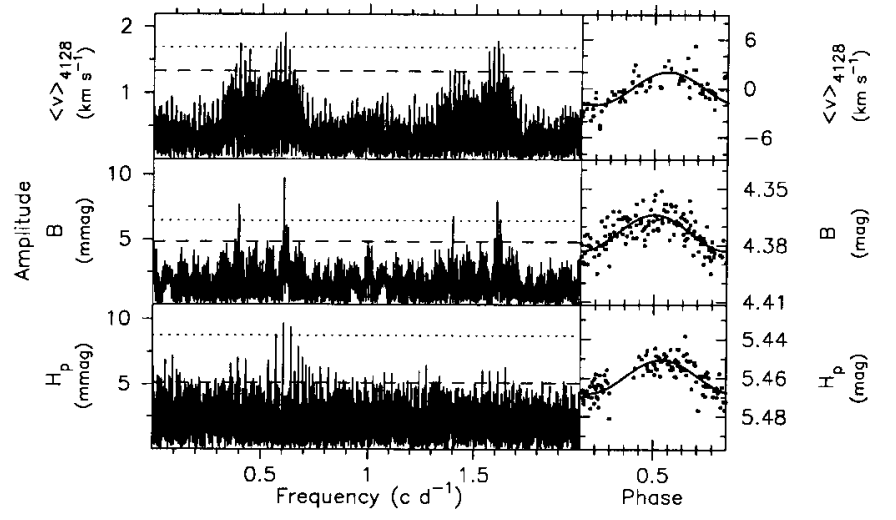

Fig. 6. Same as Fig. 2, but for the accepted intrinsic frequency $v_{1}=$ $0.6054(6) \mathrm{c} \mathrm{d}^{-1}$ of HD 53921.

Table 6. Same as Table 2, but for the accepted intrinsic frequency of HD 53921.

\begin{tabular}{ccccccc}
\hline \hline \multicolumn{2}{c}{ data-set } & $\sigma$ & $A_{i}$ & $\phi_{i}$ & $\sigma_{\text {res }}$ & $\sigma_{N}$ \\
\hline$v_{1}=0.6054(6) \mathrm{c} \mathrm{d}^{-1}$ & & & & & \\
$\langle v\rangle_{4128}$ & $\left(\mathrm{~km} \mathrm{~s}^{-1}\right)$ & 1.91 & $2.01(23)$ & $0.66(2)$ & 1.33 & 1.3 \\
$B$ & $(\mathrm{mmag})$ & 9.2 & $9.6(8)$ & $0.25(1)$ & 6.4 & \\
$H_{\mathrm{p}}$ & $(\mathrm{mmag})$ & 8.7 & $8.7(7)$ & $0.19(2)$ & 5.4 & 5.2 \\
\hline
\end{tabular}

of these two visual components is a spectroscopic binary with an eccentric orbit of about $340 \mathrm{~d}$. This suggestion also gives a natural explanation for the discrepancy between the observed $E W$ values and the effective temperature of HD 53921 (Fig. 1).

The mass/inclination procedure applied to the " $4132.5 \AA$ " orbit leads to a lower limit of $25^{\circ}$ for $i$ and of $0.65 M_{\odot}$ for $M_{2}$. The spectra were shifted according to the "Si II" orbit in an attempt to be able to study the intrinsic variability of HD 53921 afterwards.

All our data-sets of HD 53921 agree upon the first intrinsic frequency: $v_{1}=0.6054(6) \mathrm{c} \mathrm{d}^{-1}$ (Fig. 6). After prewhitening with $v_{1}$, none of the frequency peaks in the modified Scargle periodograms reach the $1 \%$ FAP-level nor the $3.7 \mathrm{~S} / \mathrm{N}$-level. This is not surprising, since $\sigma_{\text {res }}$ is already close to $\sigma_{N}$ in all data-sets. In Table 6, we give an overview of the characteristics of $v_{1}$.

\subsubsection{HD 74560 - HR 3467 - HIP 42726}

HD $74560\left(m_{V}=4.82\right)$ is one of the brightest objects of the IC 2391 cluster which is situated at about $150 \mathrm{pc}$ and is approximately $3.63 \times 10^{7}$ years old (Mermilliod 1981). In our spectra, HD 74560 reveals itself as a single-lined spectroscopic binary with an eccentric orbit of 8.378(1) days (Paper I). The application of the mass/inclination procedure leads to a lower limit on $i$ and $M_{2}$ of respectively $10^{\circ}$ and $0.25 M_{\odot}$.

HD 74560 is an SPB prototype for which Waelkens (1991) detected two well established photometric pulsation frequencies: $v_{1, \mathrm{p}}=0.64472 \mathrm{c} \mathrm{d}^{-1}$ and $v_{2, \mathrm{p}}=0.60772 \mathrm{c} \mathrm{d}^{-1}$. Two candidates for a third intrinsic frequency were also given, but the physical one could not be determined due to severe aliasing.
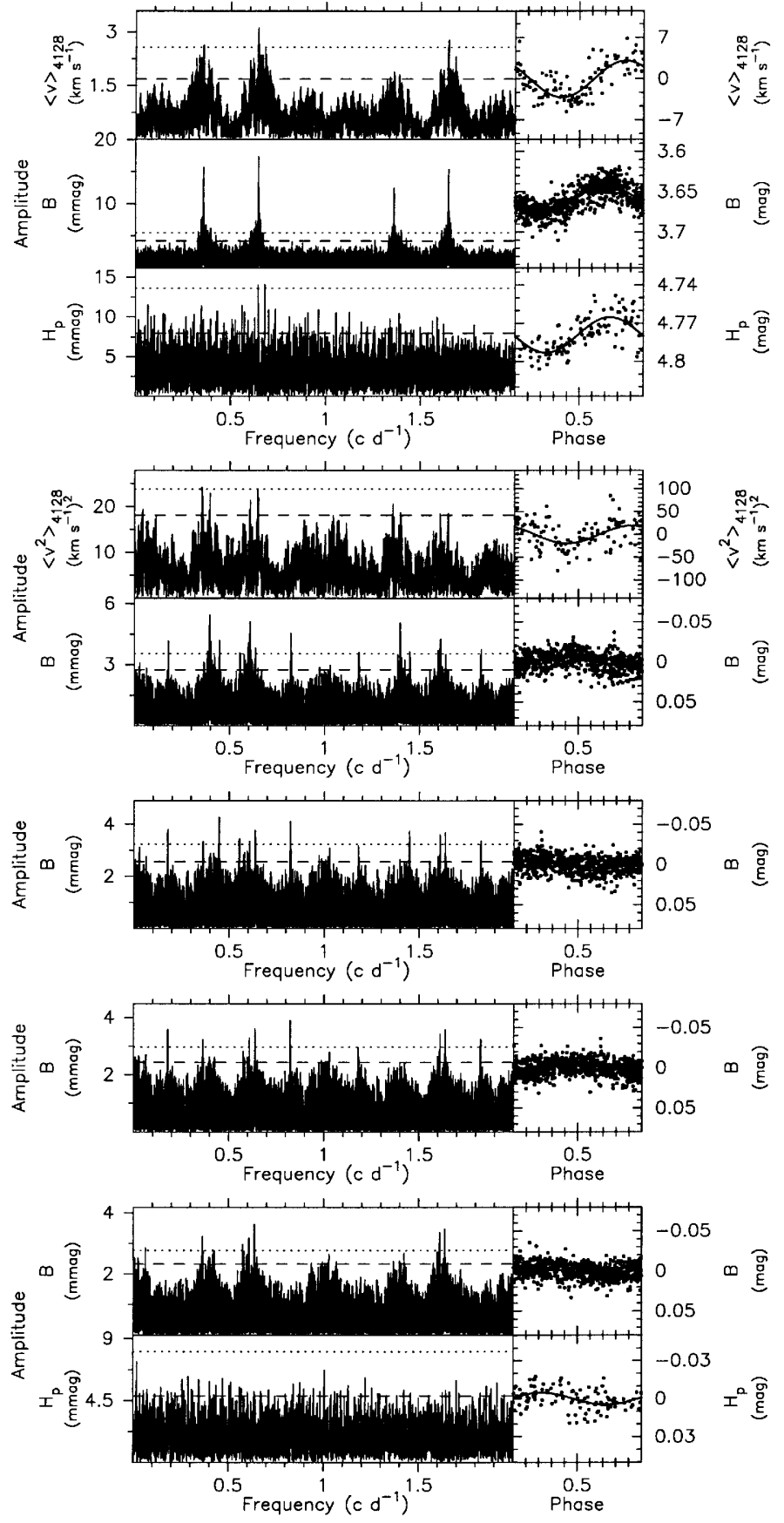

Fig. 7. Same as Fig. 2, but for the 5 accepted intrinsic frequencies $v_{1}=0.64472(9) \mathrm{cd}^{-1}, v_{2}=0.39578(9) \mathrm{cd}^{-1}, v_{3}=0.44763(9) \mathrm{cd}^{-1}$, $v_{4}=0.82281(9) \mathrm{cd}^{-1}$, and $v_{5}=0.63567(9) \mathrm{cd}^{-1}$ of HD 74560. For $v_{2}$, $\left\langle v^{2}\right\rangle$ is given instead of $\langle v\rangle$.

Although the different data-sets of HD 74560 lead to periodograms and $\theta$-statistics showing very prominent alias peaks, they all result in the same first intrinsic frequency, $v_{1}=$ $0.64472(9) \mathrm{c} \mathrm{d}^{-1}$ (Fig. 7). After prewhitening the different datasets with $v_{1}$, four additional frequencies are clearly found in the residual variations in the seven Geneva filters: $v_{2}=$ $0.39578(9) \mathrm{c} \mathrm{d}^{-1}, v_{3}=0.44763(9) \mathrm{c} \mathrm{d}^{-1}, v_{4}=0.82281(9) \mathrm{c} \mathrm{d}^{-1}$, and $v_{5}=0.63567(9) \mathrm{cd}^{-1}$. Apart from $v_{1}$, none of the frequencies coincides with those found by Waelkens (1991), although $v_{2}$ is close to the $1 \mathrm{c} \mathrm{d}^{-1}$ alias of $v_{2, \mathrm{p}}$. The sequence in which 
the frequencies is given is somewhat arbitrary since their importance in the different filters is not always the same and their amplitudes are very alike. We followed different "prewhitening" orders, but they all lead to the same set of frequencies. $v_{6}=0.60778(9) \mathrm{c} \mathrm{d}^{-1}$ and $v_{6}{ }^{\prime}=1.60510(9) \mathrm{c} \mathrm{d}^{-1}$ are found as the best candidates for the sixth intrinsic frequency in the Geneva variations after having prewhitened with $v_{1}, \ldots, v_{5}$. Our Geneva data do not allow us to choose as the blue filters are dominated by $v_{6}$ while the red ones by $v_{6}{ }^{\prime}$. Moreover, no evidence for either of the candidates is found in the other data-sets. Note that Waelkens (1991) found $v_{6}$ as second frequency in the subset he had at his disposal. Although we do not include $v_{6}$ for interpretations, we propose it as the best candidate for the sixth intrinsic frequency.

In the HIPPARCOS data there are a lot of frequencies with an amplitude exceeding only the $1 \%$ FAP-level after prewhitening with $v_{1}$. The three best candidates, 0.0197(6) $\mathrm{cd}^{-1}$, $0.0520(6) \mathrm{c} \mathrm{d}^{-1}$, and 0.0844(6) $\mathrm{c} \mathrm{d}^{-1}$, have longer periods than the expected pulsation periods in SPBs, but they can all be connected with $v_{\text {orb }}=0.11936(1) \mathrm{cd}^{-1}$ by peaks in the window function. We therefore prewhitened the residual $H_{\mathrm{p}}$ data with a fourier fit including $v_{\text {orb }}$ and its first harmonic. However, the signal at $0.0197(6) \mathrm{cd}^{-1}$ does not disappear (Fig. 7, lower panel). Besides this unexplained low frequency, one of the many frequencies with an amplitude exceeding the $1 \%$ FAPlevel is $0.6359(6) \mathrm{c} \mathrm{d}^{-1}$, which is equal to $v_{5}$ within the frequency resolution. After prewhitening with $v_{5}$, there are still several frequencies with a similar amplitude exceeding the $1 \%$ FAP-level, but no evidence for one of the candidates is found in the other data-sets. Therefore, we are not able to determine a third intrinsic frequency in the $H_{\mathrm{p}}$ data of HD 74560.

After having prewhitened the velocity moments with $v_{1}$, the best candidates in $\langle v\rangle,\left\langle v^{2}\right\rangle$ and $\left\langle v^{3}\right\rangle$ do not coincide. For $<v^{2}>, 0.3961(6) \mathrm{c} \mathrm{d}^{-1}$ and 0.6069(6) $\mathrm{c} \mathrm{d}^{-1}$ are found in the list of the frequencies with an amplitude exceeding the $1 \%$ FAPlevel (Fig. 7, second panel from top). They are close to respectively $v_{2}$ and $v_{6} . v_{6}$ does not fit the residual second moment with an acceptable quality, while a pulsation model with $v_{2}$ has the same quality as the best candidates of $\left\langle v^{2}\right\rangle$. We therefore accept $v_{2}$ to be present in the moments. After prewhitening $\left\langle v^{2}\right\rangle$ with $v_{2}$, no additional frequencies can be retained. In Table 7, we give an overview of the characteristics of the five accepted frequencies. For $v_{1}$ and $v_{5}$ to be members of the same frequency multiplet, the rotational frequency $v_{\text {rot }}$ should be close to $0.01 \mathrm{c} \mathrm{d}^{-1}$, leading to an equatorial rotation velocity of only $1.6 \mathrm{~km} \mathrm{~s}^{-1}$. This is hard to reconcile with the total line broadening of about $45 \mathrm{~km} \mathrm{~s}^{-1}$ (Aerts et al. 1999), as the pulsational broadening of the star is small.

\subsubsection{HD 177863 - HR $7241-$ HIP 93887}

HD $177863\left(m_{V}=6.28\right)$ is a fixed double system (Schrijver 1997). HD 177863A and HD 177863B are separated by 0.70 arcsec and they have a magnitude difference of approximately three. From now on, we drop the "A" in the name of the component A of the visual binary. The mass/inclination procedure applied to the eccentric 11.9154(9) day orbit derived in
Table 7. Same as Table 2, but for the accepted intrinsic frequencies of HD 74560 .

\begin{tabular}{|c|c|c|c|c|c|}
\hline data-set & $\sigma$ & $A_{i}$ & $\phi_{i}$ & $\sigma_{\text {res }}$ & $\sigma_{N}$ \\
\hline \multicolumn{6}{|l|}{$v_{1}=0.64472(9) \mathrm{c} \mathrm{d}^{-1}$} \\
\hline$<v>_{4128} \quad\left(\mathrm{~km} \mathrm{~s}^{-1}\right)$ & 2.90 & $2.85(27)$ & $0.36(2)$ & & \\
\hline$B \quad(\mathrm{mmag})$ & 16.30 & $16.8(4)$ & $0.038(4)$ & & \\
\hline$H_{\mathrm{p}} \quad(\mathrm{mmag})$ & 13.63 & $14.3(10)$ & $0.00(1)$ & & \\
\hline \multicolumn{6}{|l|}{$v_{2}=0.39578(9) \mathrm{c} \mathrm{d}^{-1}$} \\
\hline$<v>_{4128} \quad\left(\mathrm{~km} \mathrm{~s}^{-1}\right)$ & & $1.11(27)$ & $0.54(4)$ & 1.81 & 0.47 \\
\hline$B \quad(\mathrm{mmag})$ & & $4.8(4)$ & $0.38(1)$ & & \\
\hline \multicolumn{6}{|l|}{$v_{3}=0.44763(9) \mathrm{c} \mathrm{d}^{-1}$} \\
\hline$B \quad$ (mmag) & & $3.8(4)$ & $0.59(2)$ & & \\
\hline \multicolumn{6}{|l|}{$v_{4}=0.82281(9) \mathrm{c} \mathrm{d}^{-1}$} \\
\hline$B \quad(\mathrm{mmag})$ & & $4.1(4)$ & $0.29(2)$ & & \\
\hline (mmag) & & $4.8(10)$ & $0.34(3)$ & & \\
\hline \multicolumn{6}{|l|}{$v_{5}=0.63567(9) \mathrm{c} \mathrm{d}^{-1}$} \\
\hline$B \quad(\mathrm{mmag})$ & & $3.5(4)$ & $0.58(2)$ & 8.4 & \\
\hline (mmag) & & $6.0(8)$ & $0.55(3)$ & 6.8 & 4.7 \\
\hline
\end{tabular}

Paper I provides us a lower limit on $i$ of $35^{\circ}$ and restricts $M_{2}$ between $1-2 M_{\odot}$.

HD 177863 is one of the SPB-prototypes for which Waelkens (1991) determined two pulsation frequencies, $v_{1, \mathrm{p}}=$ $0.84068 \mathrm{~cd}^{-1}$ and $v_{2, \mathrm{p}}=0.90167 \mathrm{~cd}^{-1}$. Although we are dealing with strong aliasing, there is no doubt that $v_{1}=$ $0.84059(10) \mathrm{c} \mathrm{d}^{-1}$ is the main intrinsic frequency in the variations of our data-sets (Fig. 8). Note that $v_{1}$ is close to ten times the orbital frequency $v_{\text {orb }}=0.083925(6) \mathrm{c} \mathrm{d}^{-1}$ (Paper I). Willems \& Aerts (2002) showed that this mode is a resonantly excited $l=2$ mode through the time-varying tidal potential.

After prewhitening with $v_{1}$, there are still frequencies with an amplitude exceeding the 1\% FAP-level and 3.7 S/N-level in all data-sets. The residual variations in the velocity moments do not lead to convincing candidate frequencies. In the residuals of the Geneva data, the best candidates are $v_{2}=0.10108(10) \mathrm{c} \mathrm{d}^{-1}$, $v_{2}{ }^{\prime}=0.90166(10) \mathrm{c} \mathrm{d}^{-1}$ and $v_{3}=1.00525(10) \mathrm{c} \mathrm{d}^{-1} \cdot v_{2}^{\prime}$ corresponds to $v_{2, \mathrm{p}}$ and $v_{2}$ is its $\left(1 \mathrm{c} \mathrm{d}^{-1}+1 \mathrm{c} \mathrm{y}^{-1}-v\right)$ alias. With the Geneva photometry only, it is hard to decide upon the reality of the candidates, since their relative importance depends on the considered filter. However, $v_{2}$ is found in the $H_{\mathrm{p}}$ data and is therefore accepted as second intrinsic frequency in the photometric data-sets. Note that $v_{2}$ is much lower than the theoretically expected pulsation frequencies for SPBs. It is not related to the orbital frequency $v_{\text {orb }}$ either. The origin of this variation is therefore not clear. Finally we mention that, after additional prewhitening with $v_{2}$, the two frequencies $v_{3}$ and 2.00797(10) $\mathrm{c} \mathrm{d}^{-1}$ become the best candidates in the Geneva data. We refer to Table 8 for an overview of the characteristics of the intrinsic frequencies of HD 177863. 


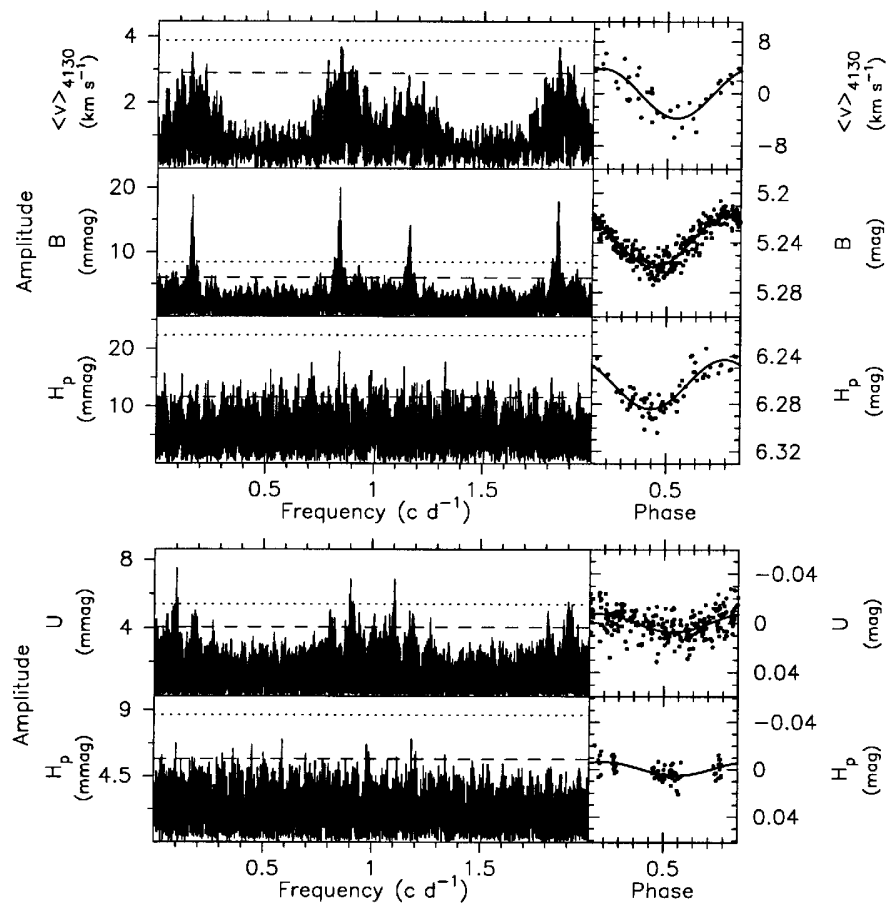

Fig. 8. Same as Fig. 2, but for the 2 accepted intrinsic frequencies $v_{1}=$ $0.84059(10) \mathrm{cd}^{-1}$, and $v_{2}=0.10108(10) \mathrm{c} \mathrm{d}^{-1}$ of HD 177863. For $v_{2}$, the Geneva $U$ filter is given instead of the Geneva $B$ filter.

Table 8. Same as Table 2, but for the accepted intrinsic frequencies of HD 177863.

\begin{tabular}{ccccccc}
\hline \hline \multicolumn{2}{c}{ data-set } & $\sigma$ & $A_{i}$ & $\phi_{i}$ & $\sigma_{\text {res }}$ & $\sigma_{N}$ \\
\hline$v_{1}=0.84059(10) \mathrm{c} \mathrm{d}^{-1}$ & & & & \\
$<v>_{4130}$ & $\left(\mathrm{~km} \mathrm{~s}^{-1}\right)$ & 3.16 & $3.82(47)$ & $0.19(2)$ & 1.89 & 1.3 \\
$B$ & $(\mathrm{mmag})$ & 15.6 & $20.6(5)$ & $0.835(4)$ & & \\
$H_{\mathrm{p}}$ & $(\mathrm{mmag})$ & 16.4 & $19.9(11)$ & $0.856(10)$ & & \\
\hline$v_{2}=0.10108(10) \mathrm{c} \mathrm{d}^{-1}$ & & & & \\
$B$ & $(\mathrm{mmag})$ & 15.6 & $3.3(5)$ & $0.68(3)$ & 6.1 & \\
$H_{\mathrm{p}}$ & $(\mathrm{mmag})$ & 16.4 & $5.9(10)$ & $0.66(4)$ & 6.5 & 6.4 \\
\hline
\end{tabular}

\subsection{Single-lined binaries with circular orbits}

\subsubsection{HD 92287 - HR 4173 - HIP 52043}

HD $92287\left(m_{V}=5.88\right)$ is a member of the Carina OBassociation (Kaltcheva 1998). Before our study, no line profile variations were reported and HD 92287 was not known as a spectroscopic binary yet. The application of the mass/inclination procedure to the 2.90457(7) day circular orbit as given in Paper I leads to a lower limit on $i$ and $M_{2}$ of respectively $30^{\circ}$ and $1.3 M_{\odot}$. At periastron, both components approach to about $10 R_{\odot}$.

HD 92287 was already known as a suspected photometric variable for 20 years (Rufener \& Bartholdi 1982). Although Waelkens \& Rufener (1985) reported $v_{\mathrm{p}}=0.6812 \mathrm{~cd}^{-1}$ as candidate frequency in their photometric data, they omitted this object from their target list. In Paper I, we showed that $v_{\mathrm{p}}$ is close to the first harmonic of the orbital frequency

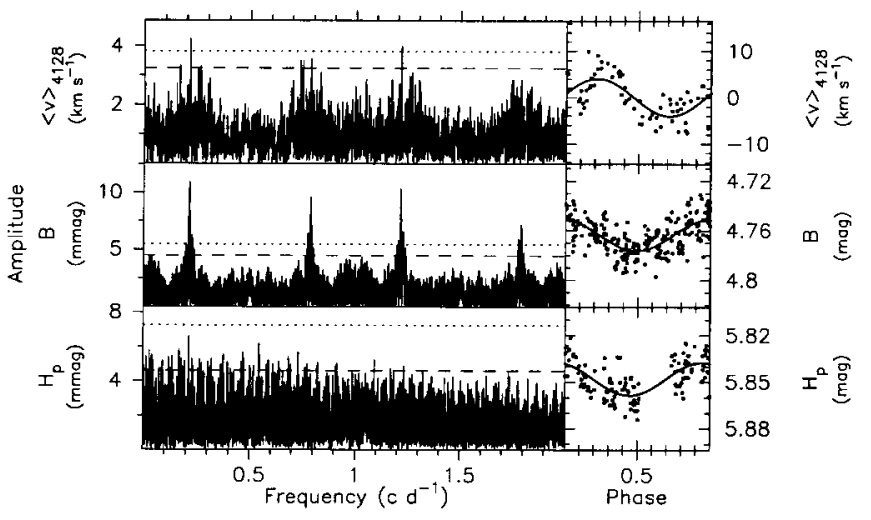

Fig. 9. Same as Fig. 2, but for the accepted intrinsic frequency $v_{1}=$ $0.21480 \mathrm{c} \mathrm{d}^{-1}$ of HD 92287 .

Table 9. Same as Table 2, but for the accepted intrinsic frequency of HD 92287.

\begin{tabular}{ccccccc}
\hline \hline \multicolumn{2}{c}{ data-set } & $\sigma$ & $A_{i}$ & $\phi_{i}$ & $\sigma_{\text {res }}$ & $\sigma_{N}$ \\
\hline$v_{1}=0.21480(7) \mathrm{c} \mathrm{d}^{-1}$ & & & & & \\
$\left\langle v>_{4128}\right.$ & $\left(\mathrm{km} \mathrm{s}^{-1}\right)$ & 4.95 & $4.85(59)$ & $0.07(2)$ & 3.41 & 2.6 \\
$B$ & $(\mathrm{mmag})$ & 13.7 & $11.3(7)$ & $0.77(1)$ & 7.1 & \\
$H_{\mathrm{p}}$ & $(\mathrm{mmag})$ & 12.0 & $7.7(9)$ & $0.79(2)$ & 6.6 & 5.5 \\
\hline
\end{tabular}

$v_{\text {orb }}=0.344236(1) \mathrm{c} \mathrm{d}^{-1}$. Therefore, we classified HD 92287 as an ellipsoidal variable.

After prewhitening the orbital variations, all data-sets point towards the same main intrinsic frequency: $v_{1}=$ 0.21480 (7) $\mathrm{c} \mathrm{d}^{-1}$ (Fig. 9). Note that $v_{1}$ is smaller than $v_{\text {orb }}$, i.e. the pulsation mode in this star has an observed pulsation period which is longer than the orbital period. The variations with $v_{1}$ in $\langle v\rangle_{4128}$ are non-sinusoidal, which is not surprising since we are dealing with a non-spherical star. After prewhitening with $v_{1}$, none of the data-sets reveal convincing candidate frequencies with an amplitude exceeding the $1 \%$ FAP-level and/or the $3.7 \mathrm{~S} / \mathrm{N}$-level. However, $\sigma_{N}$ is not reached yet by $\sigma_{\text {res }}$ in any of our data-sets. In Table 9, an overview of the characteristics of the accepted intrinsic frequency is given.

\section{Single SPBs}

We now present the results of the frequency procedure for the single stars.

\subsection{Confirmed SPBS}

\subsubsection{HD 74195 - HR 3447 - HIP 42536}

HD $74195\left(m_{V}=3.63\right)$ is one of the seven SPB prototypes, for which five intrinsic frequencies were reported by Waelkens (1991), i.e. $v_{1, \mathrm{p}}=0.35751 \mathrm{~cd}^{-1}, v_{2, \mathrm{p}}=0.35035 \mathrm{~cd}^{-1}, v_{3, \mathrm{p}}=$ $0.39856 \mathrm{~cd}^{-1}, v_{4, \mathrm{p}}=0.36048 \mathrm{~cd}^{-1}$, and $v_{5, \mathrm{p}}=0.22624 \mathrm{~cd}^{-1}$. Our data-sets reveal at least four intrinsic periods: $v_{1}=$ $0.35745(9) \mathrm{c} \mathrm{d}^{-1}, v_{2}=0.35033(9) \mathrm{c} \mathrm{d}^{-1}, v_{3}=0.34630(9) \mathrm{c} \mathrm{d}^{-1}$, and $v_{4}=0.39864(9) \mathrm{cd}^{-1}$ (Fig. 10). $v_{3}$ was not detected before. Variations with $v_{1}$ and $v_{2}$ are present in all our data-sets. 


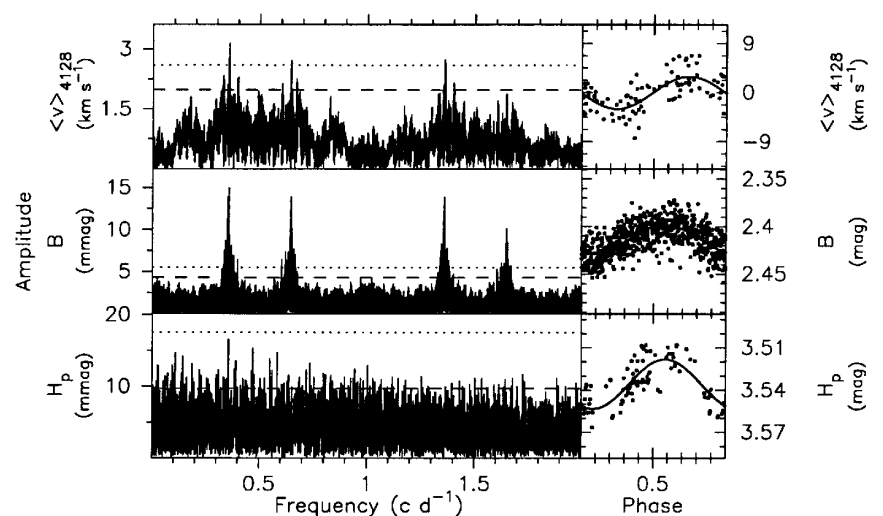

Table 10. Same as Table 2, but for the accepted intrinsic frequencies of HD 74195 .

\begin{tabular}{|c|c|c|c|c|c|}
\hline data-set & $\sigma$ & $A_{i}$ & $\phi_{i}$ & $\sigma_{\text {res }}$ & $\sigma_{N}$ \\
\hline \multicolumn{6}{|l|}{$v_{1}=0.35475(9) \mathrm{c} \mathrm{d}^{-1}$} \\
\hline$<v>_{4128}\left(\mathrm{~km} \mathrm{~s}^{-1}\right)$ & 3.14 & $3.16(21)$ & $0.48(1)$ & & \\
\hline$B \quad(\mathrm{mmag})$ & 16.6 & $15.1(5)$ & $0.168(5)$ & & \\
\hline$H_{\mathrm{p}} \quad(\mathrm{mmag})$ & 15.3 & 18.3(9) & $0.170(7)$ & & \\
\hline \multicolumn{6}{|l|}{$v_{2}=0.35033(9) \mathrm{c} \mathrm{d}^{-1}$} \\
\hline$<v>_{4128} \quad\left(\mathrm{~km} \mathrm{~s}^{-1}\right)$ & & $2.00(23)$ & $0.13(2)$ & & \\
\hline$B \quad(\mathrm{mmag})$ & & $12.2(5)$ & $0.868(6)$ & & \\
\hline$H_{\mathrm{p}} \quad(\mathrm{mmag})$ & & $11.0(7)$ & $0.883(13)$ & 5.2 & 3.4 \\
\hline \multicolumn{6}{|l|}{$v_{3}=0.34630(9) \mathrm{c} \mathrm{d}^{-1}$} \\
\hline$<v>_{4128} \quad\left(\mathrm{~km} \mathrm{~s}^{-1}\right)$ & & $1.58(21)$ & $0.64(2)$ & & \\
\hline$B \quad(\mathrm{mmag})$ & & $3.8(5)$ & $0.22(2)$ & & \\
\hline \multicolumn{6}{|l|}{$v_{4}=0.39864(9) \mathrm{c} \mathrm{d}^{-1}$} \\
\hline$<v>_{4128}\left(\mathrm{~km} \mathrm{~s}^{-1}\right)$ & & $1.32(24)$ & $0.35(3)$ & 1.35 & 0.45 \\
\hline$B \quad(\mathrm{mmag})$ & & $3.0(5)$ & $0.95(3)$ & 8.8 & \\
\hline
\end{tabular}

with $v_{1}, \ldots, v_{4}, 1.25291(9) \mathrm{c} \mathrm{d}^{-1}$ is the only frequency whose amplitude reaches the 1\% FAP-level in the Geneva data. We propose it as a candidate frequency which still needs further observational verification.

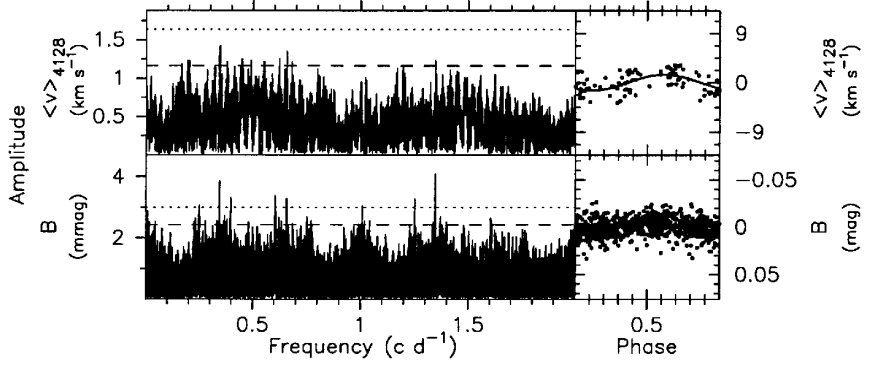

In Table 10, we give an overview of the characteristics of the frequencies, which have similar values. We find $v_{2}-v_{3}=$ $0.00403 \mathrm{c} \mathrm{d}^{-1}$ and $v_{1}-v_{2}=0.00712 \mathrm{c} \mathrm{d}^{-1} \approx 2\left(v_{2}-v_{3}\right)$, which suggests that $v_{1}, v_{2}$, and $v_{3}$ are members of a multiplet with $l \geq 2$. This suggestion would be compatible with a rotational frequency $v_{\text {rot }} \approx 0.004 \mathrm{c} \mathrm{d}^{-1}$ and hence an equatorial rotation velocity of only $0.9 \mathrm{~km} \mathrm{~s}^{-1}$. This is very small but not in contradiction with the moderate total line broadening (Aerts et al.

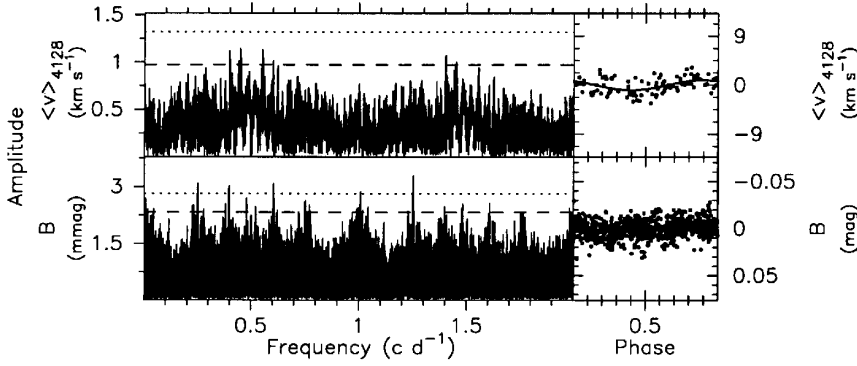
1999) as there is considerable pulsational broadening in this star.

\subsubsection{HD 181558 - HR 7339 - HIP 95159}

HD $181558\left(m_{V}=6.24\right)$ is one of the seven SPB prototypes discussed by Waelkens (1991). The 154 data points he had at his disposal already revealed three intrinsic frequencies: $v_{1, \mathrm{p}}=$ $0.80783 \mathrm{~cd}^{-1}, v_{2, \mathrm{p}}=0.41761 \mathrm{~cd}^{-1}$, and $v_{3, \mathrm{p}}=0.47365 \mathrm{~cd}^{-1}$. However, $v_{2, \mathrm{p}}$ and $v_{3, \mathrm{p}}$ could not be distinguished without ambiguity from their $(1-v)$ aliases. Our data-sets of HD 181558 are all dominated by variations with $v_{1}=0.80780(10) \mathrm{cd}^{-1}$ (Fig. 11). $v_{1}$ corresponds to $v_{1, \mathrm{p}}$ as found by Waelkens (1991). This frequency already reduces $70 \%$ up to $95 \%$ of the variance in our different data-sets.

After prewhitening the data in the Geneva filters with $v_{1}$, ble to identify $v_{3}$ and $v_{4}$ unambiguously with the Geneva data only. Indeed, evidence for the reality of $v_{3}$ and $v_{4}$ is found in the velocity variations. After prewhitening with $v_{1}$ and $v_{2}, v_{3}$ becomes the best candidate in $\langle v\rangle$ and $\left\langle v^{3}\right\rangle$. In the original $<v^{2}>$-data, the peaks of $v_{2}$ and $v_{4}$ are equally strong. Variations with $v_{1}$ only become important after prewhitening with one of these two frequencies. After subsequent prewhitening with $v_{2}$ and $v_{1}$, variations with $v_{4}$ are still present. Variations with $v_{3}$ and $v_{4}$ are not found in the $H_{\mathrm{p}}$ data. After prewhitening the search algoritms lead to two aliasses, $v_{2}=0.80721(10) \mathrm{c} \mathrm{d}^{-1}$ and $v_{2}^{\prime}=0.80760(10) \mathrm{cd}^{-1}$, whose amplitude is five times smaller than the amplitude $v_{1}$. Their relative importance depends on the considered filter. None of the two is found in the subset of Waelkens (1991), nor in our other data-sets. Both candidates are very close to $v_{1}$. This might indicate that the main frequency varies in time or that we are dealing with two real frequencies that were not yet separable in the data-set of 


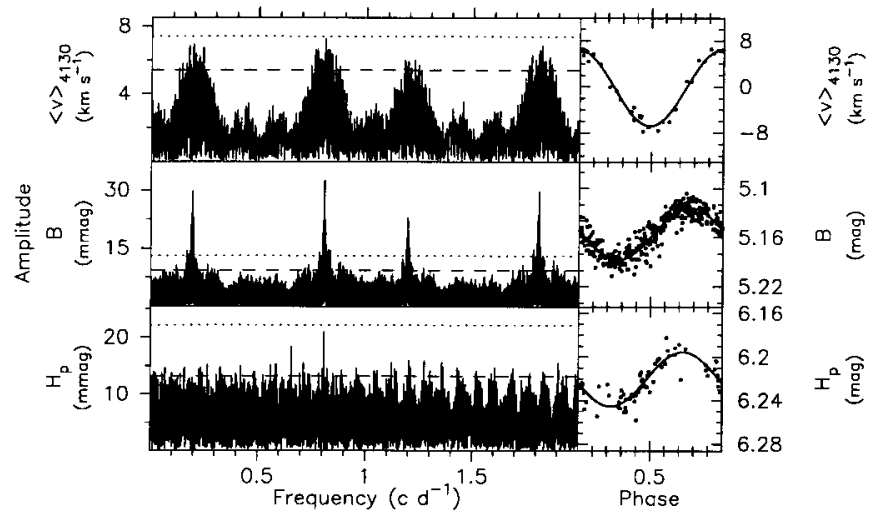

Fig. 11. Same as Fig. 2, but for the accepted intrinsic frequency $v_{1}=$ $0.80780(10) \mathrm{c} \mathrm{d}^{-1}$ of HD 181558.

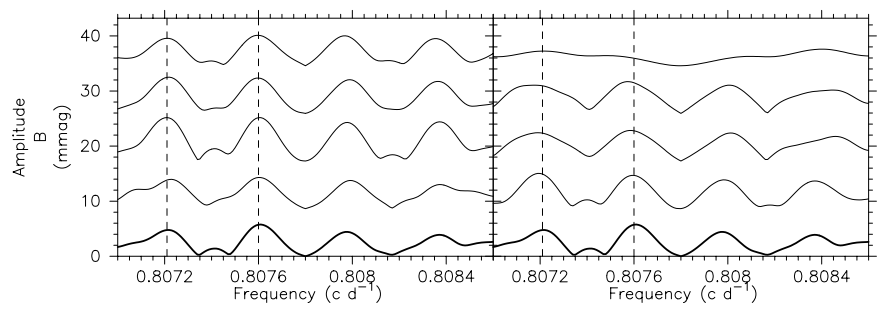

Fig. 12. Comparison of the Scargle periodogram of the Geneva $B$ filter of HD 181558 after prewhitening with $v_{1} .0 .80721 \mathrm{~cd}^{-1}$ and $0.80760 \mathrm{~cd}^{-1}$ are indicated by dashed lines. The bold periodogram corresponds to the original data-set with a time-span of $T^{*}=5544 \mathrm{~d}$. Left: Periodograms corresponding to sub-sets for which $T^{*}$ remains the same. Right: Periodograms corresponding to sub-sets with a decreasing time-span $T^{*}$ from bottom to top of respectively $5321 \mathrm{~d}$, $4903 \mathrm{~d}, 4569 \mathrm{~d}$ and $1459 \mathrm{~d}$. The latter corresponds to the data-set used by Waelkens (1991).

Waelkens (1991). Since the frequency separation between $v_{1}$ and $v_{2}^{\prime}$ is very close to $1 / T^{*} \approx 0.00018 \mathrm{c} \mathrm{d}^{-1}$ (Table 2 of Paper I $)^{2}$, the exact value of these candidate frequencies should be treated with care (Loumos \& Deeming 1978). We investigated Scargle periodograms of sub-sets composed by omitting the Geneva $B$ data of one or more observation runs after prewhitening with $v_{1}$. The position of the peaks in the modified Scargle periodogram does not change if the time-span of the sub-set remains the same as the time-span of the original data-set, while omitting an observation run at the beginning and/or the end of the original data-set does lead to minor shifts in frequency (Fig. 12). If the time-span is small enough, the frequency peaks corresponding to $v_{2}$ and $v_{2}{ }^{\prime}$ disappear. Note that all the modified Scargle periodograms shown in Fig. 12 are symmetric around $v_{1}$, although the frequency peaks corresponding to the higher-frequencies side have a somewhat lower power. If the original data-set is prewhitened with a main frequency $v_{1}{ }^{\prime}$ which slightly differs from $v_{1}$, the periodogram remains symmetric around the prewhitened frequency, but the frequency peak closest to $v_{1}$ becomes higher.

\footnotetext{
${ }^{2}$ We prefered to use $T^{*}$, the total time-span of the dedicated SPBruns in days, instead of $T$, the total time-span of all measurements in days, since the isolated first few measurements are less relevant here.
}

Table 11. Same as Table 2, but for the accepted intrinsic frequencies of HD 181558.

\begin{tabular}{ccccccc}
\hline \hline \multicolumn{2}{c}{ data-set } & $\sigma$ & $A_{i}$ & $\phi_{i}$ & $\sigma_{\text {res }}$ & $\sigma_{N}$ \\
\hline$v_{1}=0.80780(10) \mathrm{c} \mathrm{d}^{-1}$ & & & & \\
$\left\langle v>_{4130}\right.$ & $\left(\mathrm{km} \mathrm{s}^{-1}\right)$ & 5.31 & $6.64(21)$ & $0.259(6)$ & 0.85 & 0.18 \\
$B$ & $(\mathrm{mmag})$ & 25.2 & $31.9(8)$ & $0.979(4)$ & 10.2 & \\
$H_{\mathrm{p}}$ & $(\mathrm{mmag})$ & 17.7 & $24.9(19)$ & $0.924(10)$ & 9.2 & 6.2 \\
\hline
\end{tabular}

Although we cannot decide upon the reality of $v_{2}$ and $v_{2}{ }^{\prime}$, we did continue the frequency analysis in order to be able to test the significance of $v_{2, \mathrm{p}}$ and $v_{3, \mathrm{p}}$. Two different prewhitening schemes were applied: (1) After prewhitening with $v_{2}$, $v_{3}=0.80182(10) \mathrm{cd}^{-1}$ and $v_{4}=0.58617(10) \mathrm{cd}^{-1}$ are found. They are aliasses of respectively $v_{2}^{\prime}$ and $v_{2, \mathrm{p}}$. (2) After prewhitening with $v_{2}{ }^{\prime}, v_{3}{ }^{\prime}=0.80724(10) \mathrm{cd}^{-1} \approx v_{2}$ and $v_{4}{ }^{\prime}=$ $0.58615(10) \mathrm{c} \mathrm{d}^{-1} \approx v_{4}$ are found. In both cases, indications for $0.47356(10) \mathrm{c} \mathrm{d}^{-1}$ are found afterwards. Both sets of frequencies, $\left\{v_{1}, v_{2}, v_{3}, v_{4}\right\}$ and $\left\{v_{1}, v_{2}{ }^{\prime}, v_{3}{ }^{\prime}, v_{4}{ }^{\prime}\right\}$, reduce the variance by the same amount. With our current data-set, we cannot decide upon the reality of $v_{2}$ nor $v_{2}{ }^{\prime}$, and hence upon the reality of the other candidates.

After prewhitening the $H_{\mathrm{p}}$ data with $v_{1}$, the residual standard deviation $\sigma_{\text {res }}=9.2 \mathrm{mmag}$ is still large and points towards multi-periodicity. However, no other intrinsic frequencies can be determined without ambiguity. The Scargle periodograms of the velocity moments after prewhitening with $v_{1}$ do not contain amplitudes reaching the $1 \%$ FAP-level. An overview of the characteristics of the accepted intrinsic frequency is given in Table 11

\subsection{HIPPARCOS SPBS}

\subsubsection{HD 26326 - HR 1288 - HIP 19398}

Before the launch of the HIPPARCOS mission, HD 26326 $\left(m_{V}=5.43\right)$ was known as a suspected variable. Thanks to our data-sets, the photometric and spectroscopic variability of HD 26326 is beyond any doubt. Most of our data-sets of HD 26326 lead to the same first intrinsic frequency: $v_{1}=$ $0.5338(8) \mathrm{cd}^{-1}$ (Fig. 13, upper panel). Only for $\left\langle v^{2}\right\rangle$, the presence of $v_{1}$ is not clear, but no other frequency is present anyway.

After prewhitening the $H_{\mathrm{p}}$ data with $v_{1}$, many peaks in the modified Scargle periodogram still exceed the $1 \%$ FAPlevel (Fig. 13, middle panel). The three best overall candidates resulting from the periodograms and $\theta$-statistics are $1.4458(8) \mathrm{c} \mathrm{d}^{-1}, 0.1723(8) \mathrm{c} \mathrm{d}^{-1}$ and 0.5090(8) $\mathrm{c} \mathrm{d}^{-1}$, but it is hard to decide upon their reality. Combining these results with those of $\langle v\rangle$, however, allows us to conclude that $v_{2}=$ $0.1723(8) \mathrm{c} \mathrm{d}^{-1}$ is the true second intrinsic frequency since the amplitudes of $\langle v\rangle$ are maximised by a peak at $v_{2}$ (Fig. 13, middle panel). In the Geneva photometry, no evidence for $v_{2}$ is found. We therefore accept $v_{2}$ as an intrinsic frequency in the $H_{\mathrm{p}}$ and spectroscopic data only. After additional prewhitening, no other intrinsic frequencies could be found in either of the data-sets. 


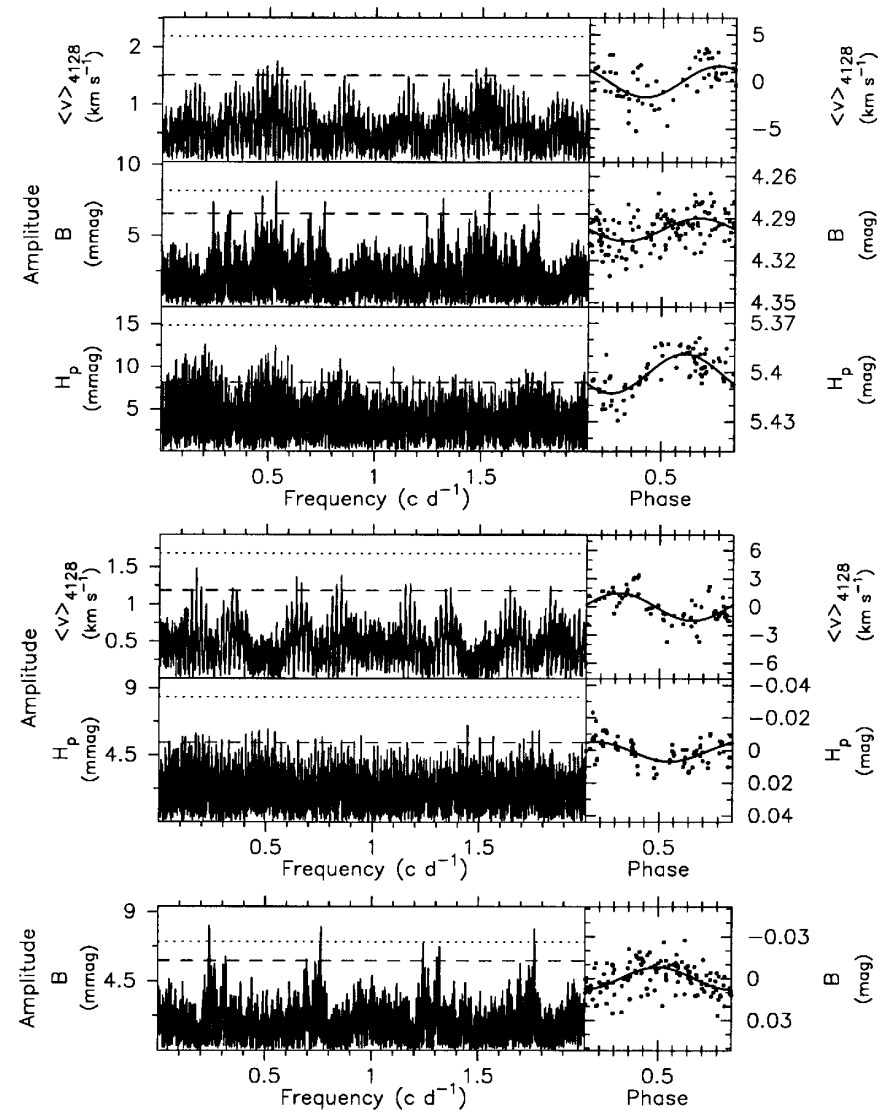

Fig. 13. Same as Fig. 2, but for the 3 accepted intrinsic frequencies $v_{1}=0.5338(8) \mathrm{cd}^{-1}, v_{2}=0.1723(8) \mathrm{cd}^{-1}$, and $v_{3}=0.7626(10) \mathrm{cd}^{-1}$ of HD 26326.

In the Geneva data, the best candidates for a second intrinsic frequency are the aliasses $0.2397(10) \mathrm{c} \mathrm{d}^{-1}$, $0.7629(10) \mathrm{cd}^{-1}$ and $1.7632(10) \mathrm{cd}^{-1}$. We do note that $0.7638(8) \mathrm{cd}^{-1}$ was one amongst many frequencies with an amplitude exceeding the 1\% FAP-level while searching for the second frequency in the $H_{\mathrm{p}}$ magnitudes. Given the frequency resolution and the totally different alias patterns of both datasets, we conclude that $v_{3}=0.7629(10) \mathrm{c} \mathrm{d}^{-1}$ is the true second intrinsic frequency in the Geneva photometry. However, further observational verification is desirable. After prewhitening with $v_{3}$, there are no longer frequencies with an amplitude reaching the $1 \%$ FAP-level in the residual Geneva variations. In Table 12, we give an overview of the characteristics of the accepted intrinsic frequencies in the different data-sets of HD 26326.

\subsubsection{HD 85953 - HR $3924-$ HIP 48527}

HD $85953\left(m_{V}=5.93\right)$ is one of our target stars for which no spectroscopic variations were detected before the start of our project. Most of our data-sets of HD 85953 agree upon the first frequency: $v_{1}=0.2663(6) \mathrm{c} \mathrm{d}^{-1}$ (Fig. 14, upper panel). In the variations of $\langle v\rangle$, also two $p$-type candidate frequencies, $8.2859(6) \mathrm{c} \mathrm{d}^{-1}$ and 9.2890(6) $\mathrm{c} \mathrm{d}^{-1}$, were found. Note that the latter is rather close to the $\beta$ Cephei-type frequency reported by Jakate (1979). These candidates, however, are both alias frequencies of $v_{1}$, from which we are tempted to conclude that
Table 12. Same as Table 2, but for the accepted intrinsic frequencies of HD 26326.

\begin{tabular}{|c|c|c|c|c|c|}
\hline data-set & $\sigma$ & $A_{i}$ & $\phi_{i}$ & $\sigma_{\text {res }}$ & $\sigma_{N}$ \\
\hline \multicolumn{6}{|l|}{$v_{1}=0.5338(8) \mathrm{c} \mathrm{d}^{-1}$} \\
\hline$<v>_{4128} \quad\left(\mathrm{~km} \mathrm{~s}^{-1}\right)$ & 2.04 & $1.80(22)$ & $0.36(2)$ & & \\
\hline$B \quad(\mathrm{mmag})$ & 12.2 & $8.9(12)$ & $0.99(2)$ & & \\
\hline$H_{\mathrm{p}} \quad(\mathrm{mmag})$ & 11.9 & $10.8(11)$ & $0.09(2)$ & & \\
\hline \multicolumn{6}{|l|}{$v_{2}=0.1723(8) \mathrm{cd}^{-1}$} \\
\hline$<v>_{4128}\left(\mathrm{~km} \mathrm{~s}^{-1}\right)$ & & $1.49(22)$ & $0.03(2)$ & 1.21 & 0.43 \\
\hline$H_{\mathrm{p}} \quad(\mathrm{mmag})$ & & $6.1(11)$ & $0.67(3)$ & 6.5 & 5.0 \\
\hline \multicolumn{6}{|l|}{$v_{3}=0.7629(10) \mathrm{cd}^{-1}$} \\
\hline$B \quad(\mathrm{mmag})$ & & $8.0(11)$ & $0.26(2)$ & 9.2 & \\
\hline
\end{tabular}
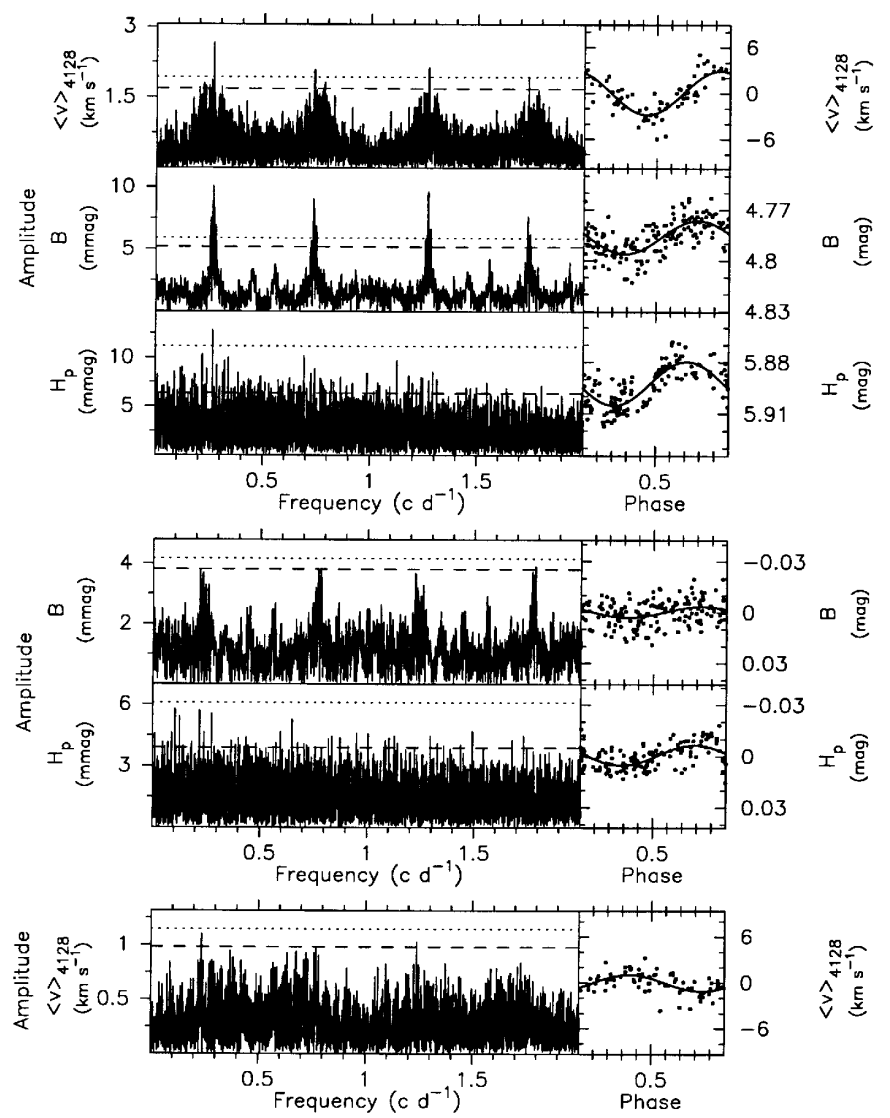

Fig. 14. Same as Fig. 2, but for the 3 accepted intrinsic frequencies $v_{1}=0.2663(6) \mathrm{c} \mathrm{d}^{-1}, v_{2}=0.2189(6) \mathrm{cd}^{-1}$, and $v_{3}=0.2353(7) \mathrm{cd}^{-1}$ of HD 85953.

the variability reported by Jakate was misinterpreted by him in terms of a $p$-mode. However, HD 85953 is situated within the common part of the instability domains of the $\beta$ Cephei stars and the SPBs (Waelkens et al. 1998). Since our current data-set is not suited for the detection of $p$-mode variability, we can not exclude that $p$ - and $g$-modes are simultaneously excited in this star.

As $v_{2}=0.2189(6) \mathrm{c} \mathrm{d}^{-1}$ is found as a candidate in the residual variations of the both the $H_{\mathrm{p}}$ magnitude and the Geneva we accept it as second frequency although the amplitude of the corresponding variations is low. After prewhitening with $v_{2}$, no 
Table 13. Same as Table 2, but for the accepted intrinsic frequencies of HD 85953.

\begin{tabular}{|c|c|c|c|c|c|}
\hline data-set & $\sigma$ & $A_{i}$ & $\phi_{i}$ & $\sigma_{\text {res }}$ & $\sigma_{N}$ \\
\hline \multicolumn{6}{|l|}{$v_{1}=0.2663(6) \mathrm{c} \mathrm{d}^{-1}$} \\
\hline$<v>_{4128}\left(\mathrm{~km} \mathrm{~s}^{-1}\right)$ & 2.35 & $2.87(21)$ & $0.31(2)$ & & \\
\hline$B \quad(\mathrm{mmag})$ & 10.3 & $10.1(8)$ & $0.97(1)$ & & \\
\hline$H_{\mathrm{p}} \quad(\mathrm{mmag})$ & 11.7 & $11.2(7)$ & $0.96(1)$ & & \\
\hline \multicolumn{6}{|l|}{$v_{2}=0.2189(6) \mathrm{c} \mathrm{d}^{-1}$} \\
\hline$B \quad(\mathrm{mmag})$ & & $3.2(8)$ & $0.92(4)$ & 7.2 & \\
\hline (mmag) & & $6.6(8)$ & $0.96(2)$ & 5.9 & 5.9 \\
\hline \multicolumn{6}{|l|}{$v_{3}=0.2353(7) \mathrm{cd}^{-1}$} \\
\hline$<v>_{4128} \quad\left(\mathrm{~km} \mathrm{~s}^{-1}\right)$ & & $1.09(19)$ & $0.90(3)$ & 1.12 & 1.1 \\
\hline
\end{tabular}

additional frequencies can be detected in the photometric observations. For the HIPPARCOS photometry, $\sigma$ is reduced to the $\sigma_{N}$ level (Table 13).

The second velocity moment $\left\langle v^{2}\right\rangle$ is the only data-set for which no signature of $v_{1}$ is found. For both Si II lines, $\left\langle v^{2}>\right.$ is dominated by $v_{3}=0.2353(7) \mathrm{cd}^{-1}$, which also turns out to be the second intrinsic frequency in $\langle v\rangle$ (Fig. 14, lower panel). $v_{3}$ is not found in the variations of the third velocity moment $\left.<v^{3}\right\rangle$. After having prewhitened $\langle v\rangle$ with $v_{1}$ and $v_{3},\left\langle v^{2}\right\rangle$ with $v_{3}$, and $\left\langle v^{3}\right\rangle$ with $v_{1}$, there are (almost) no peaks in the modified Scargle periodogram exceeding the $1 \%$ FAP-level and $\sigma_{\text {res }}$ has reached the $\sigma_{N}$ level (Table 13).

In total, three intrinsic frequencies are found for HD 85953. An overview of their characteristics is given in Table 13. The observed frequency spacings $v_{3}-v_{2}=0.0164 \mathrm{~cd}^{-1}$ and $v_{1}-$ $v_{3}=0.0310 \mathrm{c} \mathrm{d}^{-1} \approx 2\left(v_{3}-v_{2}\right)$ suggest that these frequencies are members of a multiplet with $l \geq 2$. These observed frequency spacings are indeed found if we assume a rotational frequency $v_{\text {rot }} \approx 0.02 \mathrm{c} \mathrm{d}^{-1}$, which leads to an equatorial rotation velocity of about $5 \mathrm{~km} \mathrm{~s}^{-1}$.

\subsubsection{HD 138764 - HR 5780 - HIP 76243}

HD $138764\left(m_{V}=5.15\right)$ is a bright member of the Upper Scorpius subgroup of the Scorpio-Centaurus OB-association for which Bidelman (1965) already detected asymmetries in Si II profiles. Thanks to the photometric measurements of the HIPPARCOS mission, the photometric variability of HD 138764 is beyond any doubt now. All our data-sets of HD 138764 agree upon the first intrinsic frequency $v_{1}=$ $0.7944(9) \mathrm{c} \mathrm{d}^{-1}$ (Fig. 15, upper panel). Already $80 \%$ of the variance is reduced by $v_{1}$ alone.

After prewhitening the velocity moments with $v_{1}$, the periodograms and $\theta$-statistics of $\langle v\rangle$ (Fig. 15, lower panel) and $<v^{3}>$ both point towards $v_{2}{ }^{\prime}=0.6735(9) \mathrm{c} \mathrm{d}^{-1}$. For $\left\langle v^{2}\right\rangle$, $v_{2}=0.6372(9) \mathrm{cd}^{-1}$ is found, together with other candidate frequencies. In $\left\langle v^{3}\right\rangle, v_{2}$ also reaches the $1 \%$ FAP-level. $v_{2}$ and $v_{2}^{\prime}$ are each other's alias frequencies. After having prewhitened the $H_{\mathrm{p}}$ data, the frequency search algorithms lead to $v_{2}$ (Fig. 15, lower panel) while no signature of $v_{2}{ }^{\prime}$ is found. In the residual Geneva data, there are no longer frequencies with an amplitude reaching the 1\% FAP-level. We therefore
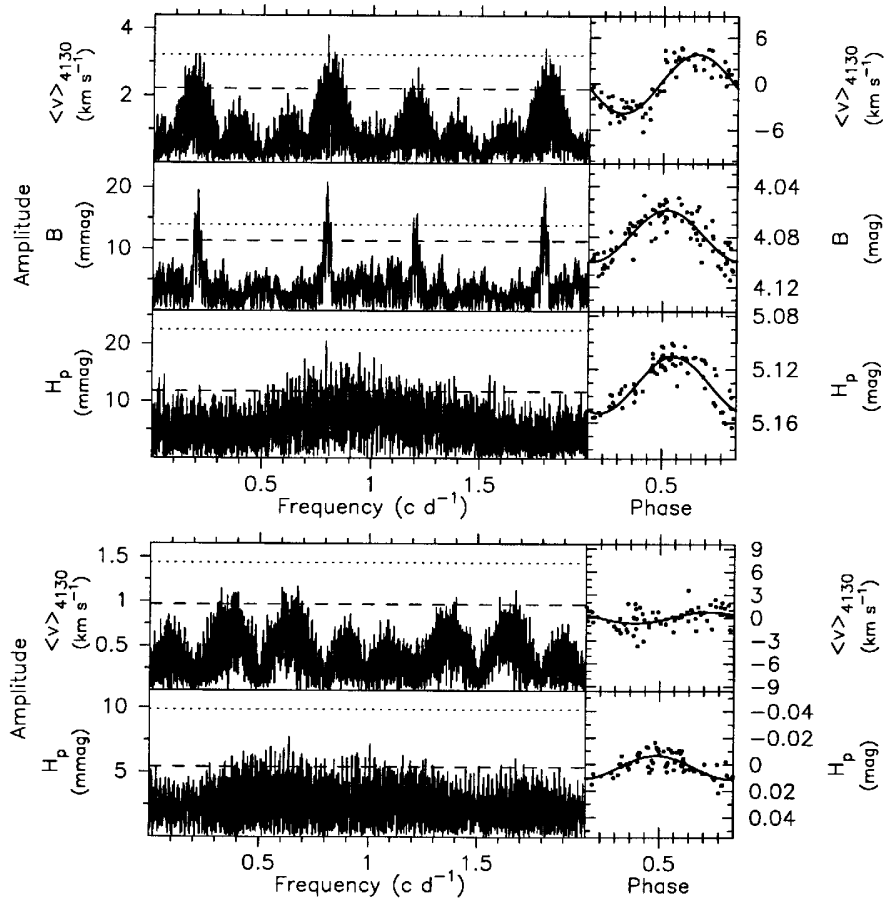

Fig. 15. Same as Fig. 2, but for the 2 accepted intrinsic frequencies $v_{1}=0.7944(9) \mathrm{c} \mathrm{d}^{-1}$, and $v_{2}=0.6372(9) \mathrm{cd}^{-1}$ of HD 138764 .

Table 14. Same as Table 2, but for the accepted intrinsic frequencies of HD 138764.

\begin{tabular}{ccccccc}
\hline \hline data-set & $\sigma$ & $A_{i}$ & $\phi_{i}$ & $\sigma_{\text {res }}$ & $\sigma_{N}$ \\
\hline$v_{1}=0.7944(9) \mathrm{c} \mathrm{d}^{-1}$ & & & & & \\
$<v>_{4130}$ & $\left(\mathrm{~km} \mathrm{~s}^{-1}\right)$ & 3.04 & $3.64(22)$ & $0.52(1)$ & & \\
$B$ & $(\mathrm{mmag})$ & 17.6 & $20.4(14)$ & $0.22(1)$ & 9.4 & \\
$H_{\mathrm{p}}$ & $(\mathrm{mmag})$ & 17.1 & $21.4(8)$ & $0.153(7)$ & & \\
\hline$v_{2}=0.6372(9) \mathrm{c} \mathrm{d}^{-1}$ & & & & & \\
$<v>_{4130}$ & $\left(\mathrm{~km} \mathrm{~s}^{-1}\right)$ & $0.80(20)$ & $0.42(5)$ & 1.20 & 0.42 \\
$H_{\mathrm{p}}$ & $\left(\mathrm{mmag}^{2}\right)$ & $9.6(9)$ & $0.27(1)$ & 5.3 & 4.4 \\
\hline
\end{tabular}

conclude that $v_{2}$ is the second physical frequency in the velocity moments and in the HIPPARCOS magnitudes. After additionally prewhitening the data-sets with $v_{2}$, no more intrinsic frequencies could be determined without ambiguity. In Table 14, we give of the characteristics of the accepted intrinsic frequencies.

\subsubsection{HD 215573 - HR 8663 - HIP 112781}

HD $215573\left(m_{V}=5.35\right)$ is one of the few normal mid-B type stars with very sharp spectral lines. It is the only star in our sample for which no obvious common main frequency is found in our different data-sets. The data in the $H_{\mathrm{p}}$ filter are dominated by two frequencies with a similar amplitude: $v_{1}=$ $0.5439(6) \mathrm{cd}^{-1}$ and $v_{2}=0.5654(6) \mathrm{c} \mathrm{d}^{-1}$ (Fig. 16, upper panel). After prewhitening with $v_{1}, v_{2}$ is found (and vice versa). For the variations in the Geneva photometry, 0.4293(9) $\mathrm{c} \mathrm{d}^{-1}$, and $0.5684(9) \mathrm{c} \mathrm{d}^{-1}$ are two of the best candidates (Fig. 16, lower panel). Both are aliasses of $v_{2}$. The frequency resolution of the 

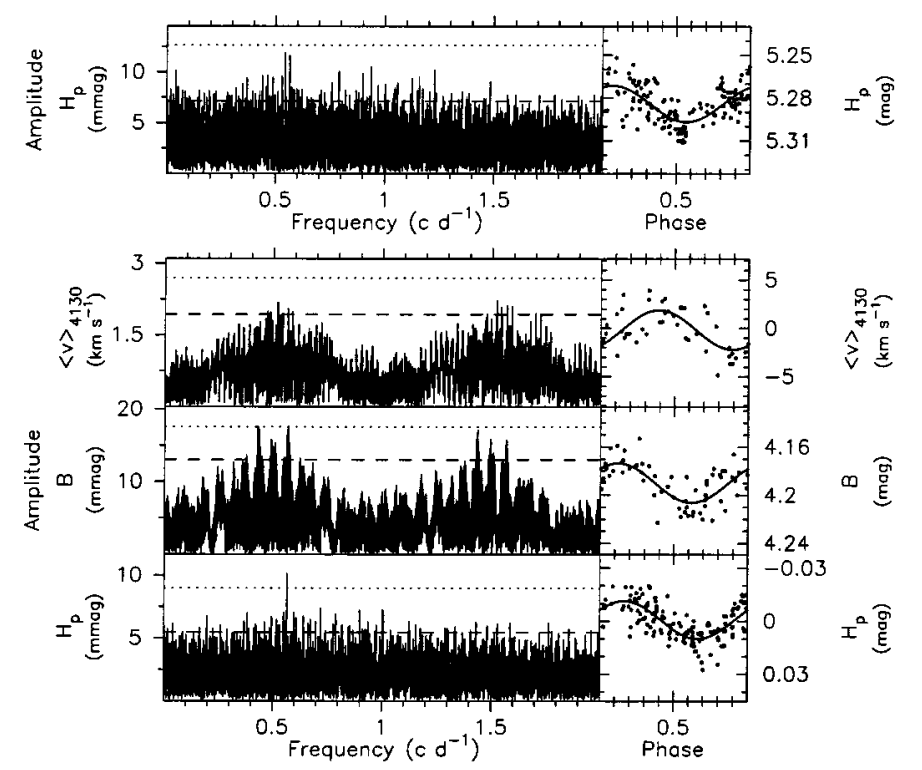

Fig. 16. Same as Fig. 2, but for the 2 accepted intrinsic frequencies $v_{1}=0.5439(6) \mathrm{cd}^{-1}$, and $v_{2}=0.5654(6) \mathrm{cd}^{-1}$ of HD 215573 .

Table 15. Same as Table 2, but for the accepted intrinsic frequencies of HD 215573.

\begin{tabular}{ccccccc}
\hline \hline \multicolumn{2}{c}{ data-set } & $\sigma$ & $A_{i}$ & $\phi_{i}$ & $\sigma_{\text {res }}$ & $\sigma_{N}$ \\
\hline$v_{1}=0.5439(6) \mathrm{c} \mathrm{d}^{-1}$ & & & & & \\
\multicolumn{2}{c}{$H_{\mathrm{p}}$} & $(\mathrm{mmag})$ & 13.2 & $11.5(10)$ & $0.68(1)$ & \\
\hline$v_{2}=0.5654(6) \mathrm{c} \mathrm{d}^{-1}$ & & & & & \\
$<v>_{4130}$ & $\left(\mathrm{~km} \mathrm{~s}^{-1}\right)$ & 2.18 & $2.03(35)$ & $0.86(3)$ & 1.61 & 0.27 \\
$B$ & $(\mathrm{mmag})$ & 17.1 & $16.3(22)$ & $0.63(2)$ & 12.1 & \\
$H_{\mathrm{p}}$ & $(\mathrm{mmag})$ & & $10.8(9)$ & $0.59(1)$ & 7.1 & 5.1 \\
\hline
\end{tabular}

43 spectra of HD 215573 is much worse than for the photometric data-sets. For $\langle v\rangle$ (Fig. 16, lower panel) and $\left\langle v^{3}\right\rangle$, the best candidate for the first intrinsic frequency is $1.521(3) \mathrm{c} \mathrm{d}^{-1}$, which is also an alias frequency of $v_{2} . v_{2}$ itself also exceeds the $1 \%$ FAP-level. Although no signature of $v_{2}$, nor of its alias frequencies is found in $\left\langle v^{2}\right\rangle$, the observed alias frequency patterns lead to the conclusion that it is commonly present in the photometric and spectroscopic data-sets. After prewhitening with $v_{2}$, the residual standard deviations of the data-sets are still quite large (Table 15). However, no other intrinsic frequencies could be determined without ambiguity. No signature of $v_{1}$ is found in the velocity moments, nor in the Geneva photometry.

In Table 15, an overview of the characteristics of the accepted intrinsic frequencies in the different data-sets of HD 215573 is given. The observed frequency spacing between $v_{1}$ and $v_{2}$ is small, which may point towards membership of frequency multiplet. For a triplet, this would imply a rotational frequency $v_{\text {rot }} \approx 0.04 \mathrm{c} \mathrm{d}^{-1}$ and an equatorial rotation velocity of some $6 \mathrm{~km} \mathrm{~s}^{-1}$, which is not incompatible with the small total line broadening (Aerts et al. 1999).
Table 16. For every star, we indicate the number of detected intrinsic frequencies in the Geneva photometric data (GEN), the HIPPARCOS photometric data (HIPP) and in the velocity moments (SPEC), and the total number (TOT). + indicates that more intrinsic frequencies are present. Confirmed SPBs known before the HIPPARCOS mission are marked with $*$.

\begin{tabular}{|c|c|c|c|c|c|c|}
\hline & & star & GEN & HIPP & SPEC & TOT \\
\hline \multirow[t]{2}{*}{$\mathrm{SB} 2 e \neq 0$} & $*$ & HD 123515 & $4+$ & 4 & 2 & $4+$ \\
\hline & & HD 140873 & 1 & 1 & 1 & 1 \\
\hline \multirow[t]{4}{*}{$\mathrm{SB} 1 e \neq 0$} & & HD 24587 & 1 & 1 & 1 & 1 \\
\hline & & HD 53921 & 1 & 1 & 1 & 1 \\
\hline & $*$ & HD 74560 & $5+$ & 3 & 2 & $5+$ \\
\hline & * & HD 177863 & 2 & 2 & 1 & 2 \\
\hline $\mathrm{SB} 1 e=0$ & & HD 92287 & 1 & 1 & 1 & 1 \\
\hline \multirow[t]{6}{*}{ Single } & $*$ & HD 74195 & $4+$ & 2 & 4 & $4+$ \\
\hline & * & HD 181558 & $1+$ & 1 & 1 & $1+$ \\
\hline & & HD 26326 & 2 & 2 & 2 & 3 \\
\hline & & HD 85953 & 2 & 2 & 2 & 3 \\
\hline & & HD 138764 & 1 & 2 & 2 & 2 \\
\hline & & HD 215573 & 1 & 2 & 1 & 2 \\
\hline
\end{tabular}

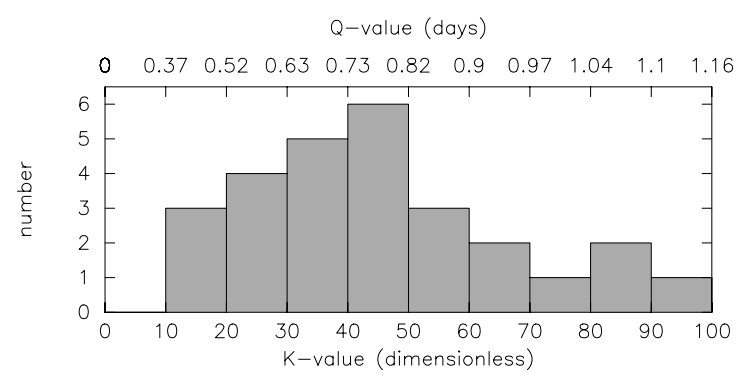

Fig. 17. The distribution of the $K$ - and $Q$-values for the observed pulsation frequencies $v$.

\section{Pulsational properties}

In Table 16, we give a summary of the results of the frequency analysis of the 13 confirmed SPBs. At least nine of them are multi-periodic. The origin of the variations of the apparent mono-periodic star HD 24587 is uncertain. In the figures of this section, the latter star is therefore indicated with a triangle while all the other considered target stars are indicated with circles.

\subsection{K- and Q-values of the observed frequencies}

In total, 30 of the observed intrinsic frequencies $v$ found in our sample are attributed to stellar pulsations. Their $K$-value is defined as the ratio of the horizontal to the vertical velocity amplitude. To a good approximation, it is given by $K \approx$ $G M / 4 \pi^{2} v^{2} R^{3}$. In Fig. 17, we give the distribution of our "observed" $K$-values, which were calculated from the mass and radius estimates given in Table 1 and from the observed frequencies. The more commonly known pulsation constant $Q$ is expressed in days and is defined as $Q \equiv \sqrt{\bar{\rho} / \bar{\rho}_{\odot}} / v$, where $\bar{\rho}$ and $\bar{\rho}_{\odot}$ denote respectively the stellar and solar average density. The $K$ - and $Q$-values are connected by the relation 


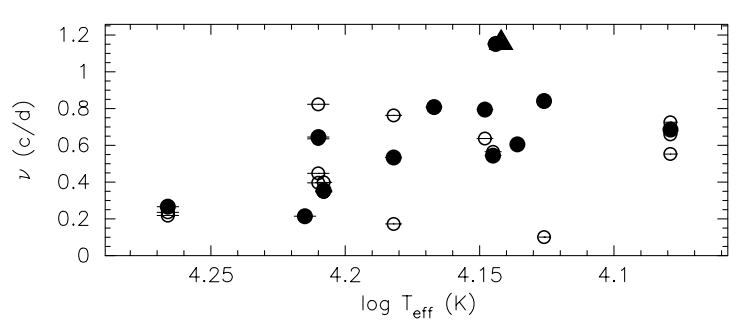

Fig. 18. The observed frequency $v\left(\mathrm{c} \mathrm{d}^{-1}\right)$ as a function of the effective temperature $T_{\text {eff }}(\mathrm{K})$. HD 24587 is indicated with a triangle while all the other considered target stars are indicated with circles. The full symbols correspond to the main frequencies.

$Q=\sqrt{K / 74.41}$. The $Q$-values are also indicated in Fig. 17 . Note that the large $K$ - and $Q$-values for $v_{1}$ of HD 92287, for $v_{2}$ of HD 26326, and for $v_{2}$ of HD 177863 are not shown for clarity. For our sample of SPBs, the distribution of the observed $K$-values is centered around 40-50. This value is higher than the one found by De Cat (2002), who studied a much larger sample of (candidate) SPBs. The SPB-pulsations are clearly all dominated by the horizontal component, which is typical for high-order $g$-mode pulsations.

\subsection{Intrinsic frequencies versus temperature}

In Fig. 18, the effective temperatures $T_{\text {eff }}$ of our SPBs are compared to their observed frequencies $v$. The observed frequencies of the coolest SPBs tend to be higher than those of the hotter stars, although the trend is only marginal. The cooler stars are situated in the lower part of the SPB instability strip, where "Maia stars" are suggested to exist (Struve 1955). However, the observed periods in our coolest targets are still much longer than those suggested for the hypothetical Maia stars.

Such a temperature-frequency relation is not expected from theoretical excitation studies. According to the $\kappa$-mechanism, the pulsation modes in SPBs can only be excited if their periods are comparable to the thermal time-scale in the Z-bump zone. This thermal time-scale decreases when $T_{\text {eff }}$ increases (Pamyatnykh 1998). Moreover, theoretical calculations show that modes with $v<0.7 \mathrm{c} / \mathrm{d}$ are damped for stars with $\log T_{\mathrm{eff}}>$ 4.2 (Dziembowski, private communication).

\subsection{Photometric versus radial velocity amplitude}

In Fig. 19, the observed amplitudes of the variations for all the observed intrinsic frequencies in the different kinds of data-sets are compared.

In the top and middle panel, the radial velocity amplitude $A A$ is compared respectively to the photometric amplitude $A_{\mathrm{B}}$ and $A_{\mathrm{H}_{\mathrm{p}}}$. For most of the observed intrinsic frequencies, a clear linear relation is found between the photometric and spectroscopic amplitudes. These linear relations are represented by the full lines denoting a least-squares linear fit (HD 92287 and HD 181558 excluded). The slopes of the fits in the upper and middle panel of Fig. 19 are respectively 5.4(8) and 4.3(7) mmag s km${ }^{-1}$. The one frequency for which the observed amplitude ratio is significantly smaller than for the
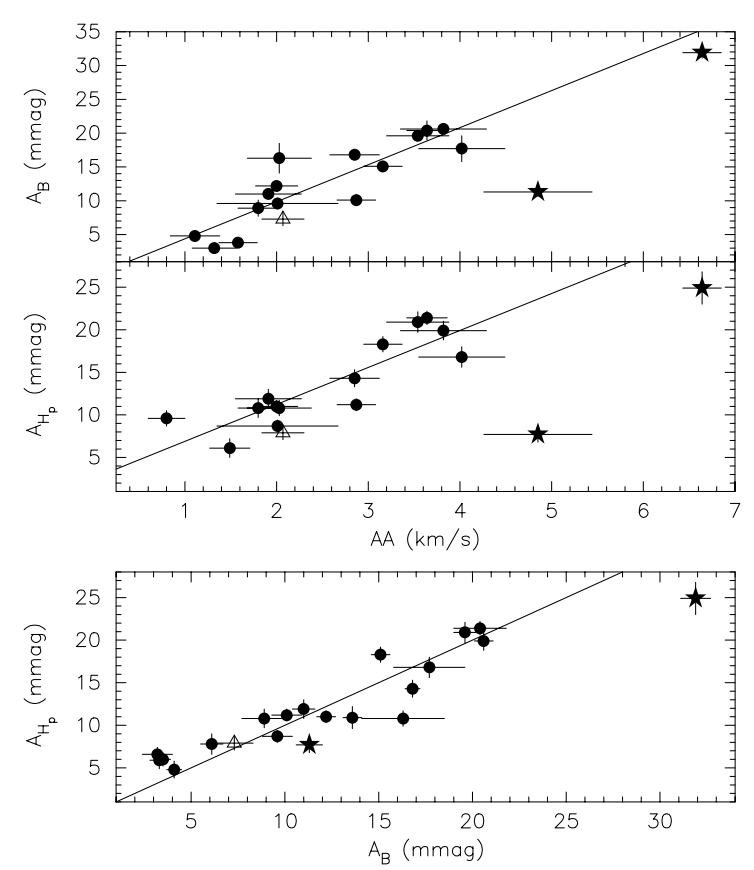

Fig. 19. Comparison of the amplitudes of variations for the observed pulsation frequencies: Geneva B versus $<v>$ (top), HIPPARCOS $H_{\mathrm{p}}$ versus $\langle v\rangle$ (middle), and HIPPARCOS $H_{\mathrm{p}}$ versus Geneva $B$ (bottom). The amplitudes for HD 24587 are marked by open triangles, those of HD 92287 and HD 181558 by full stars, and the others by full circles. The error bars denote the standard errors of the amplitudes. The full line denotes in the top and middle panel a least-squares fit the considered amplitudes (HD 92287 and HD 181558 excluded), and in the bottom panel the bisector.

others corresponds to $v_{1}$ of HD 92287 (lower star). This might indicate a high-degree mode for which one expects small photometric, but large line profile variations. Also, we recall that this star is one of the ellipsoidal variables and so is deformed due to the strong tidal forces. The observed light-to-velocity amplitude ratios are small for all our observed pulsation frequencies (Fig. 19). This seems to contradict theoretical expectations. Indeed, theoretical $5 M_{\odot}$ SPB models at different effective temperatures only reveal small light-to-velocity amplitude ratios for short pulsation periods (Dziembowski, private communication).

In the lower panel of Fig. 19, the amplitudes of the photometric data-sets are compared. The full line corresponds to an amplitude ratio $A_{H_{\mathrm{p}}} / A_{B}=1$. Only the main frequency of HD 181558 significantly deviates from the full line (upper star).

\subsection{Phase behaviour}

In Fig. 20, the observed phases of the variations for all the observed intrinsic frequencies in the different kinds of data-sets are compared. No phase lag was observed for the variations of the SPB prototypes in the data of the seven filters of the Geneva photometry (Waelkens 1991). We confirm this result for our sample, which is much larger. Phase differences in different photometric filters and/or in different colours are therefore not a good constraint to identify the modes in SPBs. 

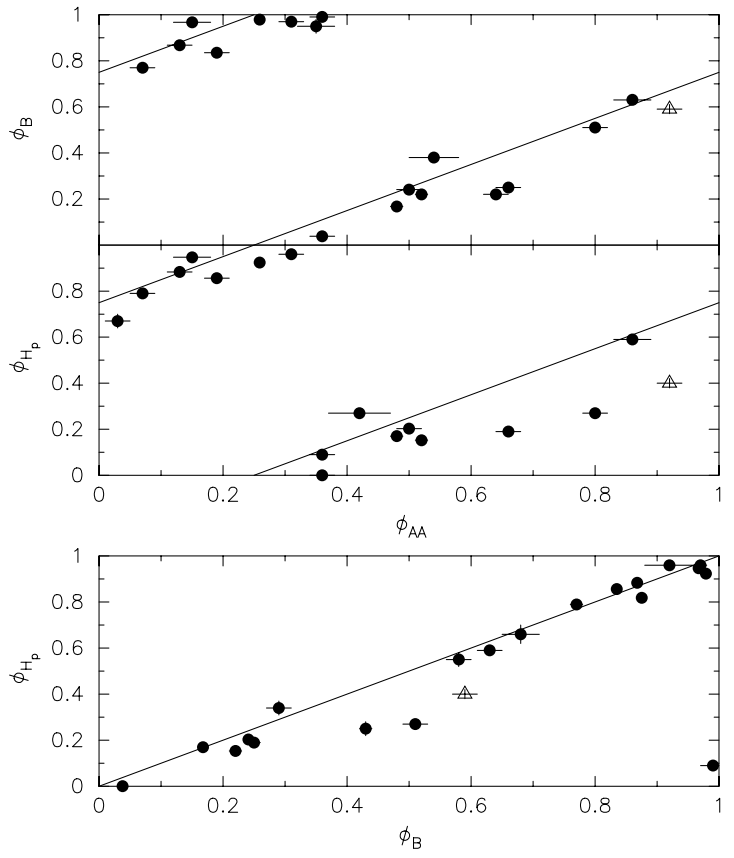

Fig. 20. Comparison of the phases of variations for the observed pulsation frequencies: Geneva $B$ versus $\langle v\rangle$ (top), HIPPARCOS $H_{\mathrm{p}}$ versus $\langle v\rangle$ (middle), and HIPPARCOS $H_{\mathrm{p}}$ versus Geneva $B$ (bottom). The phases for HD 24587 are marked by open triangles while the other targets are indicated by full circles. The error bars denote the standard errors of the phases. The full line denotes in the top and middle panel a phase lag $\Delta \phi=0.25$, and in the bottom panel $\Delta \phi=0$.

In the top and middle panel of Fig. 20, the radial velocity phases $\phi_{A A}$ are compared respectively to the photometric phases $\phi_{B}$ and $\phi_{H_{\mathrm{p}}}$. A full line corresponding to a phase lag is $\Delta \phi=0.25$ drawn. The phases of the observed pulsation variations fall within a narrow range around this line. Again, such a light-to-velocity phase behaviour is only expected theoretically for short pulsation periods (Dziembowski, private communication).

In the lower panel of Fig. 20, the photometric phases $\phi_{B}$ and $\phi_{H_{\mathrm{p}}}$ are compared. A full line is drawn for $\Delta \phi=0$. Given the absence of phase lags between the variations in the different Geneva magnitudes, we do not expect phase lags with the HIPPARCOS magnitudes either. This is generally the case for the observed variations in our SPB sample.

We stress that the frequencies displayed in Figs. 18-20 are the observed intrinsic frequencies and not the intrinsic frequencies in the corotating frame which are considered in theoretical models. Therefore, our observational results are not necessarily in contradiction with the theory.

\section{Conclusions and discussion}

Thirteen of our sample stars are now classified as confirmed SPBs and at least nine are multi-periodic. In each step of our frequency analyses, the $1 \%$ FAP-level and 3.7 S/N-level were used to judge upon the reality of different occurring frequencies. Both criteria are equivalent for multi-site data of $p$-mode pulsators (Alvarez et al. 1998). However, for our data of $g$ mode pulsators, the $3.7 \mathrm{~S} / \mathrm{N}$-level is systematically higher than the $1 \%$ FAP-level. Indeed, the mean ratio between the $1 \%$ FAPlevel and the $S / N$-level for the variations in our single-site ground-based velocity and Geneva data respectively amounts to 2.8 and 2.9, while we find a ratio of 2.2 for the variations in the continuous space-based HIPPARCOS data. We are currently investigating the sensitivity of both significance levels to time-gaps, the length of the time-base and the amount of data for typical data-sets of $g$-mode pulsators. The results of our study will also be relevant for frequency searches in $\gamma$ Doradus stars (Cuypers \& De Cat, in preparation).

In Sect. 6, we investigated for the first time the statistical properties of the SPB pulsations. Our results are not according to theoretical expectations and form a challenge for the latter. However, the frequencies used in Sect. 6 are the observed intrinsic frequencies while the intrinsic frequencies in the corotating frame are considered in theoretical models. It remains to be seen if this difference resolves the discrepancies between theoretical models and observations.

For some of our objects, we find indications for frequency multiplets. The firm confirmation of such multiplets is crucial as it is currently unclear how internal rotation behaves in such massive stars while rotational mixing has considerable influence on their internal structure and evolution (e.g., Maeder \& Meynet 2000). The study of multiplets in pulsating stars offers the possibility to constrain the internal rotation provided that we have accurate mode identifications. The latter are the topic of the next paper of this series (Paper III). First preliminary mode identifications for all the intrinsic frequencies reported in this paper with existing methods pointed towards inconsistencies between photometric and spectroscopic identifications for quite a number of cases (De Cat 2001). We judged that these methods have too limited power to identify $g$-modes. We are currently improving both types of identification methods in order to obtain more reliable and consistent results for our sample SPBs in the near future.

Acknowledgements. We are grateful to the Observatoire de Genève and to the European Southern Observatory for the generous awarding of telescope time to make this long-term project possible. Prof. W. Dziembowski and Prof. D. Kurtz are gratefully acknowledged for the constructive remarks and ideas which helped us to improve our research. We are also indebted to the referee, Dr. E. Chapellier, whose comments helped to improve the paper.

\section{References}

Aerts, C. 1996, A\&A, 314, 115

Aerts, C. 2000, A\&A, 361, 245

Aerts, C., De Pauw, M., \& Waelkens, C. 1992, A\&A, 266, 294

Aerts, C., De Cat, P., Peeters, E., et al. 1999, A\&A, 343, 872

Alvarez, M., Hernandez, M. M., Michel, E., et al. 1998, A\&A, 340, 149

Bidelman, P. W. 1965, AJ, 70, 667

Breger, M., Stich, J., Garrido, R., et al. 1993, A\&A, 271, 482

Briquet, M., De Cat, P., Aerts, C., \& Scuflaire, R. 2001, A\&A, 380, 177

Catanzaro, G., Leone, F., \& Catalano, F. A. 1999, A\&AS, 134, 211

Corbally, C. J. 1984, ApJS, 55, 657 
De Cat, P. 2001, An observational study of bright southern slowly pulsating B stars, Ph.D. Thesis, Katholieke Universiteit Leuven, Belgium

De Cat, P. 2002, in IAU Colloq. 185, Radial and Nonradial Pulsations as Probes of Stellar Physics, ed. C. Aerts, T. R. Bedding, \& J. Christensen-Dalsgaard, ASP Conf. Ser., 259, 196

De Cat, P., Aerts, C., De Ridder, J., et al. 2000, A\&A, 355, 1015 (Paper I)

Didelon, P. 1982, A\&AS, 50, 199

Dziembowski, W. A., \& Goode, P. R. 1992, ApJ, 394, 670

Dziembowski, W. A., Moskalik, P., \& Pamyatnykh, A. A. 1993, MNRAS, 265, 588

Handler, G., Breger, M., Sullivan, D. J., et al. 1996, A\&A, 307, 529

Horne, J. H., \& Baliunas, S. L. 1986, ApJ, 302, 757

Jakate, S. M. 1979, AJ, 84, 552

Kaltcheva, N. T. 1998, A\&AS, 128, 309

Leone, F., \& Catanzaro, G. 1998, A\&A, 331, 627

Loumos, G. L., \& Deeming, T. J. 1978, Astrophys. Space Sci., 56, 285

Maeder, A., \& Meynet, G. 2000, ARA\&A, 38, 143

Mathias, P., Aerts, C., Gillet, D., \& Waelkens, C. 1994, A\&A, 289, 875

Mathys, G., Manfroid, J., \& Renson, P. 1986, A\&AS, 63, 403

Mermilliod, J. C. 1981, A\&A, 97, 235
Pamyatnykh, A. A. 1998, in A Half Century of Stellar Pulsation Interpretation, A Tribute to Arthur N. Cox, ed. P. A. Bradley, \& J. A. Guzik, ASP Conf. Ser., 135, 268

Paunzen, E., Kuschnig, R., Handler, G., Gelbmann, M., \& Weiss, W. W. 1997, A\&AS, 124, 23

Renson, P., Gerbaldi, M., \& Catalano, F. A. 1991, A\&AS, 89, 429

Roberts, D. H., Lehar, J., \& Dreher, J. W. 1987, AJ, 93, 968

Rufener, F., \& Bartholdi, P. 1982, A\&AS, 48, 503

Scargle, J. D. 1981, ApJS, 45, 1

Schrijver, H. (ed.) 1997, The Hipparcos and Tycho Catalogues vol. 10, Hipparcos Double and Multiple Systems Annes, Solar System Objects (ESA Publications Division, c/o ESTEC, Noordwijk, The Netherlands)

Smith, M. A. 1977, ApJ, 215, 574

Stellingwerf, R. F. 1978, ApJ, 224, 953

Struve, O. 1955, Sky \& Telescope, 14, 461

Taylor, B. J. 1986, ApJS, 60, 577

Waelkens, C. 1991, A\&A, 246, 453

Waelkens, C., \& Rufener, F. 1985, A\&A, 152, 6

Waelkens, C., Aerts, C., Kestens, E., Grenon, M., \& Eyer, L. 1998, A\&A, 330, 215

Willems, B., \& Aerts, C. 2002, A\&A, 384, 441 